\title{
CP VIOLATION AT BABAR AND BELLE
}

\author{
Soeren Prell * \\ Iowa State University \\ Department of Physics and Astronomy \\ Ames, Iowa 50011- 3160
}

\begin{abstract}
The BABAR and Belle experiments study $C P$ violation in the $B$ meson system. Recent measurements on direct and time-dependent $C P$ asymmetries in $B$ decays are reviewed.
\end{abstract}

Presented at the 31st SLAC Summer Institute on Particle Physics: Cosmic Connection (SSI 2003), 28 Jul - 8 Aug 2003, Menlo Park, CA

*Supported in part by the Department of Energy Grant DE-FG02-01ER41155 and Contract DE-AC03-76SF00515Stanford Linear Accelerator Center, Stanford University, Stanford, CA 94309 


\section{Contents}

1 Introduction 2

1.1 CKM Matrix and Unitarity Triangle . . . . . . . . . . . . . . 2

1.2 Time-independent CP Asymmetries . . . . . . . . . . . . . . . . 4

1.3 Time-dependent CP Asymmetries . . . . . . . . . . . . . . . . . 4

2 Experimental Techniques 6

2.1 The $B$ Factories PEP-II and KEK-B . . . . . . . . . . . . . . . . 6

2.2 The BABAR and Belle Detectors . . . . . . . . . . . . . 7

2.3 Reconstruction of $B \bar{B}$ Events . . . . . . . . . . . . . . . . . . . 7

$2.4 \quad B$ Flavor Tagging . . . . . . . . . . . . . . . . . 8

$2.5 \Delta t$ Measurement . . . . . . . . . . . . . . . 9

3 The Measurement of $\sin 2 \beta \quad 9$

3.1 Charmonium Modes . . . . . . . . . . . . . . 10

3.2 Cabibbo-suppressed Modes . . . . . . . . . . . . . . . 13

3.2.1 $B^{0} \rightarrow J / \psi \pi^{0} \ldots \ldots \ldots \ldots \ldots$

$3.2 .2 \quad B^{0} \rightarrow D^{* \pm} D^{\mp} \ldots \ldots \ldots \ldots 14$

3.2.3 $B^{0} \rightarrow D^{*+} D^{*-} \ldots \ldots \ldots \ldots$

3.3 Penguin-Dominated Modes . . . . . . . . . . . . . . . 16

3.3.1 $B^{0} \rightarrow \phi K_{S}^{0} \ldots \ldots \ldots \ldots 17$

3.3.2 $B^{0} \rightarrow \eta^{\prime} K_{S}^{0} \ldots \ldots \ldots \ldots \ldots$

3.3.3 Charmonium Modes versus Penguin-dominated Modes . . . . . 19

4 Measurement sensitive to $\sin 2 \alpha \quad 19$

$4.1 \quad B^{0} \rightarrow \pi^{+} \pi^{-} \ldots \ldots \ldots \ldots \ldots$

$4.2 \quad B^{0} \rightarrow \rho^{ \pm} \pi^{\mp} \ldots \ldots \ldots \ldots \ldots \ldots$

5 Measurements sensitive to $\gamma \quad \mathbf{2 6}$

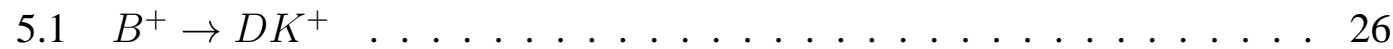

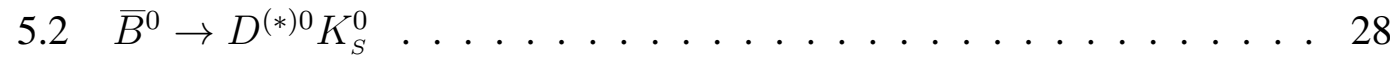

$5.3 B^{0} \rightarrow D^{(*) \mp} \pi^{ \pm} \ldots \ldots \ldots \ldots$

6 Conclusion $\quad 32$ 


\section{Introduction}

One of the most important open questions in particle physics is the origin of $C P$ violation. $C P$ violation is a necessary ingredient to create a matter-antimatter asymmetry from a symmetric matter-antimatter situation shortly after the big bang. ${ }^{1}$ However, the amount of $C P$ violation that is consistent with the current measurements in the $K$ and $B$ meson systems cannot account for the matter-antimatter asymmetry of the universe. ${ }^{2,3}$ Sources of $C P$ violation beyond the Standard Model are needed to account for that asymmetry.

According to the Standard Model, $C P$ violation in the weak interaction of quarks is described by only one $C P$-odd phase in the Cabibbo-Kobayashi-Maskawa (CKM) quark mixing matrix. The pattern of $C P$ asymmetries in weak charged current transitions resulting from the structure of the CKM matrix is thus a key prediction of the Standard Model. It is the goal of the $B$ factory experiments $B A B A R$ and Belle to test this prediction and to search for discrepancies that would provide evidence for new physics beyond the Standard Model.

\subsection{CKM Matrix and Unitarity Triangle}

$C P$ violation has been of great interest in particle physics since its discovery in 1964 in the decays of $K_{L}^{0}$ mesons. ${ }^{4}$ An elegant explanation of the origin of the observed $C P$ asymmetry was proposed by Kobayashi and Maskawa, as a complex phase in the three-generation CKM quark-mixing matrix. ${ }^{5}$ In the Standard Model with three quark generations, the CKM matrix has four independent parameters one of which breaks the $C P$ symmetry for flavor-changing charged currents. In the Wolfenstein parameterization $^{6}$ the CKM matrix $V_{\mathrm{CKM}}$ is given by

$$
V_{\mathrm{CKM}} \equiv\left(\begin{array}{ccc}
V_{u d} & V_{u s} & V_{u b} \\
V_{c d} & V_{c s} & V_{c b} \\
V_{t d} & V_{t s} & V_{t b}
\end{array}\right)=\left(\begin{array}{ccc}
1-\frac{1}{2} \lambda^{2} & \lambda & A \lambda^{3}(\rho-i \eta) \\
-\lambda & 1-\frac{1}{2} \lambda^{2} & A \lambda^{2} \\
A \lambda^{3}(1-\rho-i \eta) & -A \lambda^{2} & 1
\end{array}\right)
$$

The amplitude $A$ for a weak transition between two quarks is proportional to the corresponding CKM matrix element.

$$
A\left(q \rightarrow W q^{\prime}\right) \propto V_{q q^{\prime}}^{*}
$$

while the amplitude for the $C P$-conjugated process is proportional to its complexconjugate 


$$
A\left(\bar{q} \rightarrow W \bar{q}^{\prime}\right) \propto V_{q q^{\prime}}
$$

The CKM matrix is unitary which leads to relations between its elements. For example, the orthogonality of the first and third columns

$$
V_{u d} V_{u b}^{*}+V_{c d} V_{c b}^{*}+V_{t d} V_{t b}^{*}=0
$$

leads to the so-called "Unitarity Triangle" in the $\rho-\eta$ plane as shown in Fig. 1. The inner angles of the Unitarity Triangle are called $\alpha, \beta$, and $\gamma$ or alternatively $\phi_{2}$, $\phi_{1}$, and $\phi_{3}$, respectively. The measurements of these angles are the primary goals of the $B A B A R$ and Belle experiments. The Standard Model can be tested by measuring $\alpha$, $\beta$, and $\gamma$ in a variety of decays. The consistency between these measurements and the consistency with measurements of the sides of the Unitarity Triangle and the measured $C P$ asymmetries in kaon decays will provide a strong test of the CKM description of $C P$-violation.

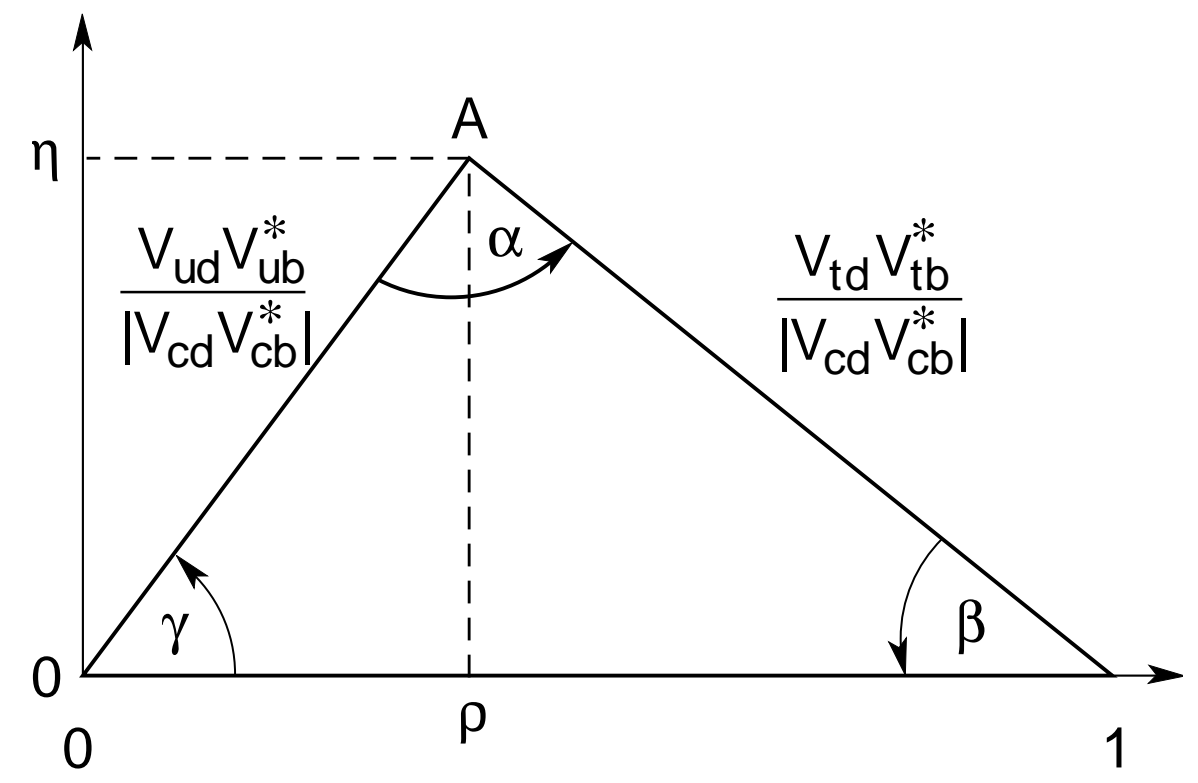

Fig. 1. The normalized Unitarity Triangle determined from the orthogonality of the first and third columns of the CKM matrix. 


\subsection{Time-independent CP Asymmetries}

The time-independent or direct $C P$ asymmetry between a $B$ decay to a final state $f$ and its $C P$-conjugate decay $\bar{B} \rightarrow \bar{f}$ is defined as

$$
A_{C P} \equiv \frac{\Gamma(B \rightarrow f)-\Gamma(\bar{B} \rightarrow \bar{f})}{\Gamma(B \rightarrow f)+\Gamma(\bar{B} \rightarrow \bar{f})} .
$$

A non-zero $A_{C P}$ can be produced, if the decay $B \rightarrow f$ can proceed via at least two amplitudes $A_{j}=\left|A_{j}\right| e^{i\left(\phi_{j}+\delta_{j}\right)}$ with different weak phases $\phi_{j}$ and different strong phases $\delta_{j}$. For example, if a decay amplitude $A$ and the corresponding $C P$-conjugate decay amplitude $\bar{A}$ can be written as the sum of two amplitudes $A_{1}$ and $A_{2}$

$$
\begin{aligned}
A & =\left|A_{1}\right| \exp \left[i\left(\delta_{1}+\phi_{1}\right)\right]+\left|A_{2}\right| \exp \left[i\left(\delta_{2}+\phi_{2}\right)\right] \\
\bar{A} & =\left|A_{1}\right| \exp \left[i\left(\delta_{1}-\phi_{1}\right)\right]+\left|A_{2}\right| \exp \left[i\left(\delta_{2}-\phi_{2}\right)\right]
\end{aligned}
$$

the resulting asymmetry is

$$
A_{C P}=\frac{2\left|A_{1}\right|\left|A_{2}\right| \sin (\Delta \delta) \sin (\Delta \phi)}{\left|A_{1}\right|^{2}+\left|A_{2}\right|^{2}+2\left|A_{1}\right|\left|A_{2}\right| \cos (\Delta \delta) \cos (\Delta \phi)} .
$$

Therefore, direct $C P$ asymmetries can only occur, if the phase differences $\Delta \delta=$ $\delta_{1}-\delta_{2}$ and $\Delta \phi=\phi_{1}-\phi_{2}$ are not zero. From the measured branching fractions, one can calculate the magnitudes of the decay amplitudes $|A|$ and $|\bar{A}|$. However, the interesting physics parameters $\left|A_{1}\right|,\left|A_{2}\right|, \Delta \delta$, and especially $\Delta \phi$ can only be obtained with additional decay modes that are related via a symmetry (e.g. isospin) and provide additional constraints on the parameters.

\subsection{Time-dependent CP Asymmetries}

A $B^{0}$ can transform itself into a $\bar{B}^{0}$ via a second order weak current box diagram such as the one shown in Fig. 2.

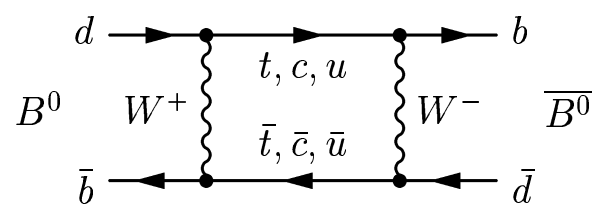

Fig. 2. Feynman diagram for $B^{0} \bar{B}^{0}$ mixing. 
This results in the phenomena of $B^{0} \bar{B}^{0}$ oscillations and time-dependent $C P$ asymmetries. ${ }^{7}$ The time-dependent amplitudes for $B^{0}$ and $\bar{B}^{0}$ mesons to a final state $f$ are given by

$$
\begin{aligned}
\left\langle f|H| B_{\text {phys }}^{0}(t)\right\rangle & =e^{-i m t} e^{-\Gamma t / 2}\left[A_{f} \cos \left(\Delta m_{B} t / 2\right)+i \frac{q}{p} \bar{A}_{f} \sin \left(\Delta m_{B} t / 2\right)\right] \\
\left\langle f|H| \bar{B}_{\text {phys }}^{0}(t)\right\rangle & =e^{-i m t} e^{-\Gamma t / 2}\left[i \frac{p}{q} A_{f} \sin \left(\Delta m_{B} t / 2\right)+\bar{A}_{f} \cos \left(\Delta m_{B} t / 2\right)\right]
\end{aligned}
$$

where $A_{f} \equiv\left\langle f|H| B^{0}\right\rangle$ is the amplitude for a $B^{0}$ decay to the final state $f$ and $\bar{A}_{f} \equiv\left\langle f|H| \bar{B}^{0}\right\rangle$ is the corresponding amplitude for the $\bar{B}^{0}$ decay. The coefficients $p$ and $q$ transform the $B^{0}$ and $\bar{B}^{0}$ states into the weak eigenstates: $\left|B_{L, H}^{0}\right\rangle=p\left|B^{0}\right\rangle \pm q\left|\bar{B}^{0}\right\rangle$. The average and difference between the $B_{H}$ and $B_{L}$ masses are $m$ and $\Delta m_{B}$, respectively, and we assume the difference between the $B_{H}$ and $B_{L}$ widths to be negligible. From the Feynman diagrams for $B^{0} \bar{B}^{0}$ mixing the ratio $(q / p)$ can be calculated. The virtual top quark dominates the loop and

$$
\frac{q}{p}=\frac{V_{t b}^{*} V_{t d}}{V_{t b} V_{t d}^{*}}
$$

which in the Wolfenstein phase convention is $e^{-i 2 \beta}$. The time-dependent decay rate to a final state $f$ then becomes

$$
\Gamma_{ \pm}(t)=\frac{e^{-t / \tau}}{4 \tau}\left\{1 \pm S_{f} \sin \left(\Delta m_{B} t\right) \mp C_{f} \cos \left(\Delta m_{B} t\right)\right\}
$$

where the $+(-)$ sign indicates that the $B$ was produced as a $B^{0}\left(\bar{B}^{0}\right)$. The coefficients $S_{f}$ and $C_{f}$ in front of the time-dependent sine and cosine terms are given by

$$
S_{f} \equiv \frac{2 \mathcal{I} m \lambda_{f}}{1+\left|\lambda_{f}\right|^{2}} \quad C_{f} \equiv \frac{1-\left|\lambda_{f}\right|^{2}}{1+\left|\lambda_{f}\right|^{2}}
$$

where

$$
\lambda_{f}=(q / p) \bar{A}_{f} / A_{f}
$$

For the two simple cases of $B^{0} \bar{B}^{0}$ mixing and $B$ decays to a $C P$ eigenstate through one or more decay amplitudes with the same weak phase the time-dependent decay rates can immediately be obtained. If a $B^{0}$ decays to a flavor specific final state via a single amplitude $A_{f}$, while $\bar{A}_{f}=0$, such as the semi-leptonic decays $B^{0} \rightarrow D^{(*)-} l^{+} \nu$, 
we have $\lambda=0$ and consequently $S_{f}=0$ and $C_{f}=1$. In this case, we obtain the $\left(C P\right.$-conserving) time-dependent decay rate for $B^{0} \bar{B}^{0}$ oscillations:

$$
\Gamma_{ \pm}(t)=\frac{e^{-t / \tau}}{4 \tau}\left\{1 \mp \cos \left(\Delta m_{B} t\right)\right\}
$$

On the other hand for a decay to a $C P$ eigenstate that occurs with a single weak phase such as $B \rightarrow J / \psi K_{S}^{0}$ the decay amplitudes are of the same magnitude $\left|A_{f}\right|=\left|\bar{A}_{f}\right|$ and $\left|\lambda_{f}\right|=1$. In this case, $C_{f}=0$ and $S_{f}=\mathcal{I} m \lambda_{f}$ and the time-dependent decay rates are given by

$$
\Gamma_{ \pm}(t)=\frac{e^{-t / \tau}}{4 \tau}\left\{1 \mp \mathcal{I} m \lambda_{f} \sin \left(\Delta m_{B} t\right)\right\}
$$

The time-dependent decay rates for modes which proceed through at least two amplitudes with different weak phases are typically more complex.

\section{Experimental Techniques}

\subsection{The $B$ Factories PEP-II and KEK-B}

At the asymmetric-energy $e^{+} e^{-}$colliders PEP-II at SLAC and KEK-B at KEK, resonant production of $\Upsilon(4 S)$ mesons provides a copious source of $B \bar{B}$ pairs. The $\Upsilon(4 S)$ are produced at PEP-II (KEK-B) with a boost $\beta \gamma=0.56$ (0.43) along the collision axis, with nominal energies of 9.0 (8.0) and 3.1 (3.5) $\mathrm{GeV}$ for the electron and positron beams, respectively. The $B$ mesons produced from the decay $\Upsilon(4 S) \rightarrow B \bar{B}$ are nearly at rest in the $\Upsilon(4 S)$ frame $\left(p^{*} \approx 325 \mathrm{MeV} / c\right)$ and the proper decay-time difference between the two $B$ decays is to a good approximation proportional to the distance between the $B$ decay vertices along the boost direction ( $z$-axis), $\Delta t \approx \Delta z /(c \beta \gamma)$. The average value of $|\Delta z|$ is $\beta \gamma c \tau_{B^{0}} \approx 250 \mu \mathrm{m}(200 \mu \mathrm{m})$ at PEP-II (KEK-B).

The BABAR and Belle experiments have been collecting $e^{+} e^{-}$collision data since 1999. At the time of the 2003 summer shutdowns the BABAR and Belle experiments had accumulated $131 \mathrm{fb}^{-1}$ and $159 \mathrm{fb}^{-1}$, respectively. Most of this data was taken on the $\Upsilon(4 S)$ resonance while about $10 \%$ was taken $40-60 \mathrm{MeV}$ below the $\Upsilon(4 S)$ resonance ("off-resonance" sample). The latter is used to study $e^{+} e^{-} \rightarrow q \bar{q}(q=u, d, s, c)$ continuum backgrounds. 


\subsection{The BABAR and Belle Detectors}

The BABAR and Belle experiments are state-of-the-art multi-purpose detectors optimized for the study of $B$ decays. Charged particles are detected and their momenta measured by a combination of a silicon vertex tracker and a central drift chamber, in a 1.5-T solenoidal field. The average vertex resolution in the $z$ direction is about $70 \mu \mathrm{m}$ for a fully reconstructed $B$ meson. Electrons and photons are identified by a CsI electromagnetic calorimeter. Muons are identified in muon chambers. In $B A B A R$, a Cherenkov ring imaging detector covering the central region, together with $\mathrm{d} E / \mathrm{d} x$ information from the drift chamber and the silicon tracker provides $K-\pi$ separation of at least three standard deviations for $B$ decay products with momentum greater than $250 \mathrm{MeV} / c$ in the laboratory. The Belle particle identification system includes an aero-gel Cherenkov counter and a time-of-flight system and can distinguish kaons from pions up to momenta of $3.5 \mathrm{GeV} / c$ with $90 \%$ efficiency and a fake rate of less than $5 \%$. Detailed descriptions of the $B A B A R$ and Belle detectors can be found elsewhere. ${ }^{8,9}$

\subsection{Reconstruction of $B \bar{B}$ Events}

For most of the analyses presented here, the decay of one $B$ meson is fully reconstructed from its charged and neutral daughter particles in a certain final state $f$. The usual selection criteria for fully reconstructed $B$ decays are the energy difference $\Delta E$ between the energy of the reconstructed $B$ candidate and the beam energy in the $\Upsilon(4 S)$ frame and the beam-energy substituted mass $m_{\mathrm{ES}}$ :

$$
\Delta E=E_{B}^{*}-E_{\text {beam }}^{*}, \quad m_{\mathrm{ES}}=\sqrt{E_{\text {beam }}^{*}-\left|\vec{p}_{B}^{*}\right|^{2}}
$$

where the asterisk denotes the $\Upsilon(4 S)$ center-of-mass frame. The signal distributions of these variables peak at $\Delta E=0$ and $m_{\mathrm{ES}}=m_{B}$. Typical resolutions are $\sigma\left(m_{\mathrm{ES}}\right)=$ $3 \mathrm{MeV} / c^{2}$ and $\sigma(\Delta E)=15-50 \mathrm{MeV}$.

The reconstruction of $B$ decays to charmless final states suffers from large combinatorial background from $e^{+} e^{-} \rightarrow q \bar{q}(q=u, d, s, c)$ interactions. On average continuum background events exhibit a more jet-like structure while $B \bar{B}$ events are more isotropic in the center-of-mass frame. $B A B A R$ and Belle exploit this in their analyses of charmless $B$ decays by using event topology-based discriminating variables including the angle between the thrust axis of the $B$ candidate and the remaining tracks and energy clusters in the event and the energy flow in the event with respect to the $B$ candidate thrust axis. 
In some analyses multiple discriminating variables are used in a likelihood fit or combined into a Fisher discriminant or a neural network to increase the separation between signal decays and background.

The other $B$ in the event is not fully reconstructed. However, for time-dependent analyses of neutral $B$ decay we need to know its decay point and whether it is a $B^{0}$ or a $\bar{B}^{0}$. The decay products of the fully reconstructed $B \rightarrow f$ candidate are removed from the event and the remaining particles are examined to determine the decay vertex and flavor of the other $B$.

\section{$2.4 \quad B$ Flavor Tagging}

After the daughter tracks of the fully reconstructed $B, B_{\text {rec}}$, are removed from the event, the remaining tracks are analyzed to determine the flavor tag $\left(B^{0}\right.$ or $\left.\bar{B}^{0}\right)$ of the other $B, B_{\text {tag }}$. For this purpose, flavor tag information carried by leptons from semileptonic $B$ decays, charged kaons, soft pions from $D^{*}$ decays, and high momentum charged particles is examined. $B A B A R^{10}$ uses neural networks, trained to each of the physics processes described above, and classifies events into four mutually exclusive categories according to the underlying physics process, combined with performance criteria based on the neural net output. Belle ${ }^{11}$ uses the likelihood ratios of the properties of the charged particles to estimate the mistag rate for each individual event, and then ranks events into six categories based on their estimated mistag rate.

The amplitudes of the observed time-dependent $C P$ asymmetries are reduced by a factor $1-2 w$, where $w$ is the flavor mistag rate. Both experiments determine the mistag rates of the various tagging categories from data. This is done by studying decays to flavor-specific final states for which the deviation of the observed mixing asymmetry from unity is given by $1-2 w$. BABAR uses fully reconstructed events in the modes $B^{0} \rightarrow D^{(*)-} h^{+}\left(h^{+}=\pi^{+}, \rho^{+}, a_{1}^{+}\right)$and the mode $B^{0} \rightarrow J / \psi K^{* 0}\left(K^{* 0} \rightarrow K^{+} \pi^{-}\right)$. Belle uses fully reconstructed events in the modes $B^{0} \rightarrow D^{(*)-} h^{+}\left(h^{+}=\pi^{+}, \rho^{+}\right)$and the mode $B^{0} \rightarrow D^{*-} l^{+} \nu$.

Despite the differences between the flavor tagging algorithms of the two experiments, the performance is very similar: the total effective tagging efficiency $Q=\sum_{i} \epsilon_{i}(1-$ $\left.2 w_{i}\right)^{2}$, where $\epsilon_{i}$ is the fraction of events in tagging category $i$ and the sum is over all tagging categories, is measured to be $(28.1 \pm 0.7) \%$ for $B A B A R$ and $(28.8 \pm 0.6) \%$ for Belle. 


\section{5 $\Delta t$ Measurement}

If a $B^{0} \bar{B}^{0}$ pair is produced in an $\Upsilon(4 S)$ decay it evolves as a coherent $P$-wave until one of the $B$ mesons decays. If the $B_{\text {tag }}$ decays to a flavor specific final state i.e. as $B^{0}$ or $\bar{B}^{0}$ at a time $t_{\text {tag }}$ then $B_{\text {rec }}$ at that time must be of the opposite flavor due to Bose symmetry. As a consequence, in equations 10, 13, and 14 that describe the time-dependent decay rates the time $t$ has to be replaced with the time-difference $\Delta t=t_{\mathrm{rec}}-t_{\mathrm{tag}}$. Here, $\Delta t$ is a signed variable and the equations for the time-dependent decay rates are also valid for negative $\Delta t$.

As $B_{\text {rec }}$ is fully reconstructed, its decay vertex position is well measured. The decay vertex of $B_{\text {tag }}$ is inferred from the charged particle tracks remaining after the decay products of $B_{\text {rec }}$ are removed from the event. To reduce biases in the $B_{\text {tag }}$ decay vertex reconstruction both experiments remove tracks from $K_{S}^{0}$ and $\Lambda$ candidates and photon conversions. In addition tracks with a large contribution to the vertex $\chi^{2}$ are removed by an iterative procedure. Belle requires that the $B_{\text {tag }}$ decay vertex is consistent with the beamspot. Instead $B A B A R$ requires the $B_{\text {tag }}$ vertex to be consistent with the line of flight computed from the location of the beamspot, the momentum of $B_{\text {rec }}$, and the $\Upsilon(4 S)$ boost.

The time difference $\Delta t$ is related to the distance $\Delta z$ between the decay vertices of $B_{\text {rec }}$ and $B_{\text {tag }}$ by $\Delta z=\beta \gamma \gamma_{\text {rec }}^{*} c \Delta t+\gamma \beta_{\text {rec }}^{*} \gamma_{\text {rec }}^{*} \cos \theta_{\text {rec }}^{*} c\left(\tau_{B}+|\Delta t|\right)$, where $\theta_{\text {rec }}^{*}, \beta_{\text {rec }}^{*}$, and $\gamma_{\mathrm{rec}}^{*}$ are the polar angle with respect to the boost direction, the velocity and the boost of $B_{\text {rec }}$ in the $\Upsilon(4 S)$ frame and the sum of the proper times of $B_{\text {rec }}$ and $B_{\text {tag }}$ has been approximated by $\tau_{B}$. BABAR solves the equation for $\Delta t$ and Belle make the approximation $\Delta t=\Delta z / c \beta \gamma \gamma_{\text {rec }}^{*}$ which keeps only the first term. The resolution in $\Delta t$ measured from the fully reconstructed sample to flavor specific final states is $1.1 \mathrm{ps}$ for $B A B A R$ and 1.4 ps for Belle, in part due to the difference in the $\Upsilon(4 S)$ boost.

\section{The Measurement of $\sin 2 \beta$}

One can qualitatively separate the $B$ decay modes sensitive to $\sin 2 \beta$ in three classes: the golden modes, the penguin-polluted modes, and the penguin-dominated modes. The golden modes consist of final states with a charmonium resonance and a kaon such as $B^{0} \rightarrow J / \psi K_{S}^{0}$ and proceed through a $b \rightarrow c \bar{c} s$ tree amplitude. These modes have a relatively large branching fraction, a clear experimental signature, and the measured $C P$ asymmetry can be related to $\sin 2 \beta$ without large theoretical uncertainties. The golden 
modes are used to determine $\sin 2 \beta$ and compare it to measurements of the sides of the Unitarity Triangle. The penguin-polluted modes are Cabibbo-suppressed and have in addition to a dominant $b \rightarrow c \bar{c} d$ tree amplitude a significant, but hard to calculate, penguin amplitude. These modes will give important information about the relative size of penguin and tree contributions. The penguin-dominated modes proceed dominantly through a $b \rightarrow s \bar{s} s$ or a $b \rightarrow s \bar{d} d$ penguin amplitude with small contributions from tree amplitudes or penguin amplitudes with different weak phases. The $C P$-asymmetries in these modes can be compared to the $C P$-asymmetry in the golden modes to search for large deviations that provide evidence for possible contributions from physics processes beyond the Standard Model.

\subsection{Charmonium Modes}

$B A B A R$ and Belle extract $\sin 2 \beta$ from samples of fully reconstructed $B^{0}$ decays $\left(B_{\mathrm{CP}}\right)$ to final states that contain a charmonium resonance and a neutral kaon such as $\bar{B}^{0} \rightarrow$ $J / \psi K_{S}^{0}$.

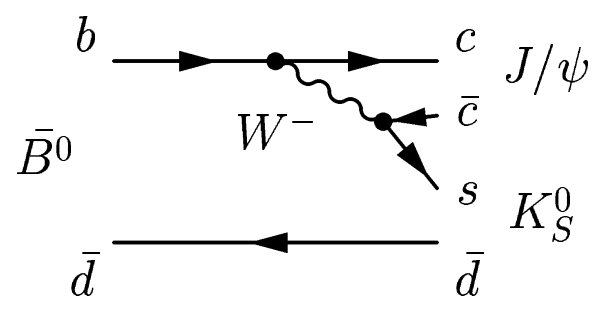

Fig. 3. Leading Feynman diagram for the decay $\bar{B}^{0} \rightarrow J / \psi K_{S}^{0}$.

The decay $\bar{B}^{0} \rightarrow J / \psi K_{S}^{0}$ proceeds through the Cabibbo-favored color-suppressed $b \rightarrow c \bar{c} s$ tree diagram shown in Fig. 3. A similar diagram exists for the $C P$-conjugate decay $B^{0} \rightarrow J / \psi K_{S}^{0}$. Therefore, a $\bar{B}^{0}$ can either decay directly to the $J / \psi K_{S}^{0}$ final state or change its flavor followed by the decay of the $B^{0}$ to the same final state. The interference between these two amplitudes results in a time-dependent $C P$ asymmetry as given by Eq. 10.

Assuming the Wolfenstein phase convention, in Eq. 12 the ratio of amplitudes $\bar{A}_{f} / A_{f}$ is given by

$$
\frac{\bar{A}_{f}}{A_{f}}=\eta_{f}\left(\frac{V_{c b} V_{c s}^{*}}{V_{c b}^{*} V_{c s}^{*}}\right)\left(\frac{q}{p}\right)_{K}=\eta_{f} \frac{V_{c b} V_{c s}^{*}}{V_{c b}^{*} V_{c s}^{*}} \frac{V_{c s} V_{c d}^{*}}{V_{c s}^{*} V_{c d}^{*}}=\eta_{f}
$$



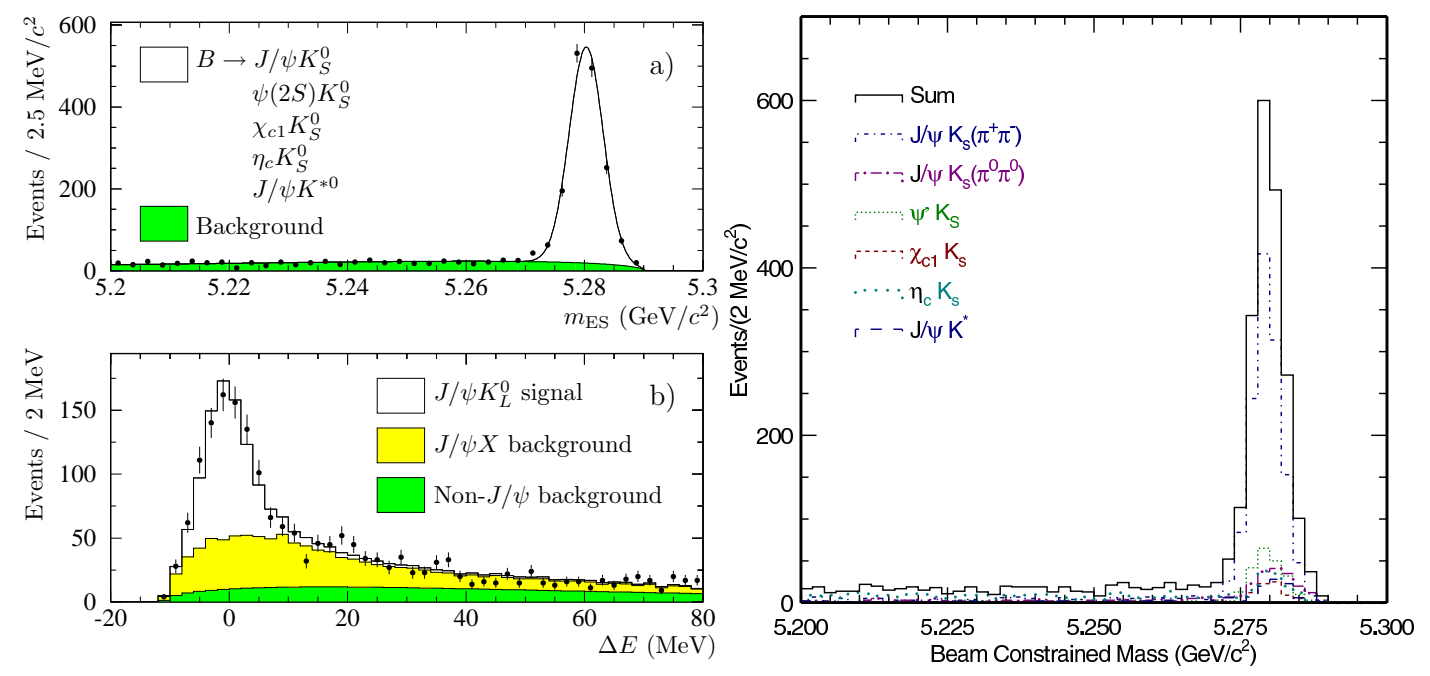

Fig. 4. Distribution of $m_{\mathrm{ES}}$ for $B_{C P}$ candidates with a $K_{S}^{0}$ in the final state from $B A B A R$ (upper left) and Belle (right); distribution of $\triangle E$ for $J / \psi K_{L}^{0}$ candidates from $B A B A R$ (lower left).

where $n_{f}=+1(-1)$ for a $C P$ even (odd) final state $f$. If we combine Eq. 9 and Eq. 16, we obtain

$$
\lambda_{f}=\left(\frac{q}{p}\right) \frac{\bar{A}}{A}=\eta_{f} e^{-2 i \beta} \Longrightarrow \mathcal{I} m \lambda_{f}=-\eta_{f} \sin 2 \beta
$$

which is phase convention independent. Thus, the time-dependent $C P$-violating asymmetry for charmonium-containing $b \rightarrow c \bar{c} s$ decays is

$$
A_{C P}(\Delta t) \equiv \frac{\Gamma_{+}(\Delta t)-\Gamma_{-}(\Delta t)}{\Gamma_{+}(\Delta t)+\Gamma_{-}(\Delta t)}=-\eta_{f} \sin 2 \beta \sin \left(\Delta m_{B} \Delta t\right) .
$$

$B A B A R^{10}$ and Belle ${ }^{11}$ have published their $\sin 2 \beta$ measurements with samples of $88 \times 10^{6}$ and $85 \times 10^{6} B \bar{B}$ events, respectively. They increase their event samples by reconstructing $B$ decays to several charmonium $K_{S}^{0}$ final states $\left(J / \psi K_{S}^{0}, \psi(2 S) K_{S}^{0}\right.$, $\left.\chi_{c 1} K_{S}^{0}, \eta_{c} K_{S}^{0}, J / \psi K^{* 0}\left(K_{S}^{0} \pi^{0}\right)\right)$ as well as $B^{0} \rightarrow J / \psi K_{L}^{0}$. The modes with a $K_{S}^{0}$ in the final state $\left(J / \psi K_{S}^{0}, \psi(2 S) K_{S}^{0}, \chi_{c 1} K_{S}^{0}, \eta_{c} K_{S}^{0}\right)$ are $C P$-odd whereas the mode $J / \psi K_{L}^{0}$ is $C P$-even. The mode $B^{0} \rightarrow J / \psi K^{* 0}$ has $C P$-even and $C P$-odd contributions to its decay rate due to the presence of even $(L=0,2)$ and odd $(L=1)$ orbital angular momenta. The experiments take this into account by using an effective $C P$-content derived from the measured contribution of the $L=1$ component. ${ }^{12}$ 
The distributions of $m_{\mathrm{ES}}$ for charmonium $K_{S}^{0}$ events are shown in Fig. 4. For the $J / \psi K_{L}^{0}$ events, only the direction of the $K_{L}^{0}$ is measured and to determine the $K_{L}^{0}$ momentum the mass of the $B^{0}$ candidate is constrained to the $B^{0}$ mass. The distribution of $\triangle E$ for the $J / \psi K_{L}^{0}$ events $(B A B A R)$ is also shown in Fig. 4.
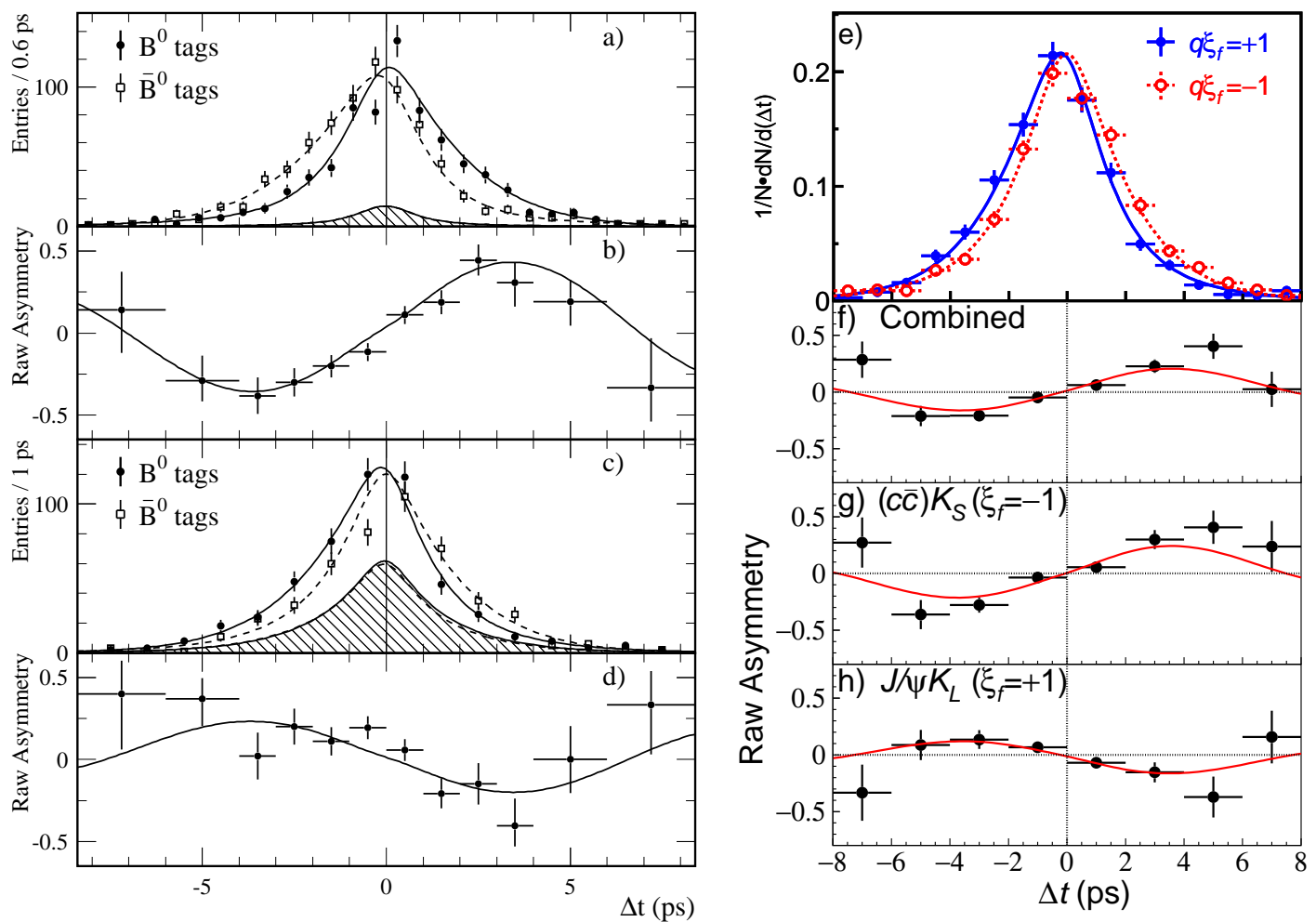

Fig. 5. Distributions of $\Delta t$ and raw flavor asymmetries for $B_{\mathrm{CP}}$ candidates from $B A B A R$ and Belle. $B A B A R$ (left): Distributions of $\triangle t$ and raw asymmetries for $B_{\mathrm{CP}}$ candidates having a $K_{S}^{0}$ (a and b) or a $K_{L}^{0}$ (c and d), respectively, in the final state. Belle (right): Raw asymmetries for $B_{\mathrm{CP}}$ events with a $K_{S}^{0}(\mathrm{~g})$ or a $K_{L}^{0}(\mathrm{~h})$, respectively, in the final state. Distributions of $\Delta t$ (e) and raw asymmetry (f) for all $B_{\mathrm{CP}}$ candidates. Events with a $B^{0}$-tag $\left(\bar{B}^{0}\right.$-tag) have $q=+1(-1)$ and $\xi_{f}$ denotes the $C P$-eigenvalue of the final state.

The value of $\sin 2 \beta$ is determined from unbinned likelihood fits to the $\Delta t$ distributions of the selected candidates. The projections of the fit results on the observed $\Delta t$ distributions and raw flavor asymmetries are shown in Fig. 5. A difference in the $\Delta t$ distributions for $B^{0}$ and $\bar{B}^{0}$ tagged events is apparent. The measured $\sin 2 \beta$ values are

$$
\begin{aligned}
& \sin 2 \beta=0.741 \pm 0.067 \pm 0.034 \quad(B A B A R), \\
& \sin 2 \beta=0.719 \pm 0.074 \pm 0.035 \quad \text { (Belle) }
\end{aligned}
$$


The measurements are in good agreement with each other and combining them yields $\sin 2 \beta=0.731 \pm 0.056 .{ }^{13}$ The constraint of this measurement on the apex of the Unitarity Triangle is shown in Fig. 6. It is in good agreement with constraints derived from measurements of $C P$-conserving $B$ decays and the observed $C P$ violation in the neutral kaon system.

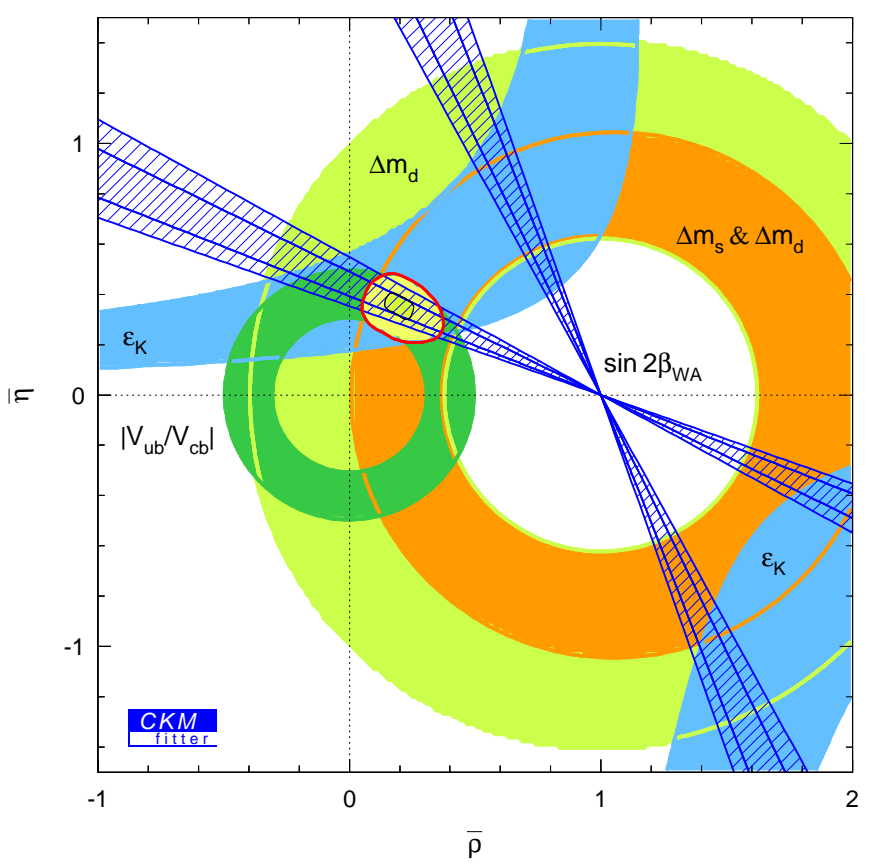

Fig. 6. Constraints on the apex of the Unitarity Triangle from indirect measurements in the $(\bar{\rho}-\bar{\eta})$-plane $\left(\bar{\rho}=\rho\left(1-\lambda^{2} / 2\right), \bar{\eta}=\eta\left(1-\lambda^{2} / 2\right)\right)$ overlaid with the direct measurement of $\sin 2 \beta$. $^{14}$

\subsection{Cabibbo-suppressed Modes}

Violations of $C P$ symmetry are also expected in decays that proceed via a Cabibbosuppressed $b \rightarrow c \bar{c} d$ amplitude (e.g. $J / \psi \pi^{0}$ and $D^{(*)+} D^{(*)-}$ ). In contrast to the $\mathcal{O}\left(\lambda^{2}\right)$ $b \rightarrow c \bar{c} s$ modes, these have tree amplitudes of $\mathcal{O}\left(\lambda^{3}\right)$. These processes are sensitive to the presence of possible new physics, because due to their smaller amplitudes interference terms can be relatively more prominent. However, these measurements are more challenging because of lower decay rates, higher backgrounds, and complications in the interpretation of the measured $C P$-asymmetries due to the simultaneous presence of tree and penguin amplitudes. 


\subsection{1 $\quad B^{0} \rightarrow J / \psi \pi^{0}$}

The decay $B^{0} \rightarrow J / \psi \pi^{0}$ proceeds dominantly through a Cabibbo-suppressed $b \rightarrow c \bar{c} d$ transition. In the Standard Model $B^{0} \rightarrow J / \psi \pi^{0}$ has a contributing penguin amplitude with the same weak phase as the tree amplitude and an additional penguin amplitude with a different weak phase. The tree amplitude and the penguin amplitude with different weak phase are equal to leading order in $\lambda$. Therefore, $B^{0} \rightarrow J / \psi \pi^{0}$ may have a $C P$ asymmetry that differs from $B^{0} \rightarrow J / \psi K_{S}^{0}$ with the deviation serving as a probe of the contribution of the penguin decay amplitude with different weak phase. $B A B A R^{15}$ and Belle ${ }^{16}$ have measured the $C P$ asymmetries in $B^{0} \rightarrow J / \psi \pi^{0}$ with $81 \mathrm{fb}^{-1}$ and $78 \mathrm{fb}^{-1}$, respectively:

$$
\begin{aligned}
& S_{J / \psi \pi^{0}}=0.05 \pm 0.49 \pm 0.16 \quad C_{J / \psi \pi^{0}}=0.38 \pm 0.51 \pm 0.09 \quad \text { (BABAR) } \\
& S_{J / \psi \pi^{0}}=-0.93 \pm 0.49 \pm 0.08 \quad C_{J / \psi \pi^{0}}=0.25 \pm 0.39 \pm 0.06 \quad \text { (Belle) }
\end{aligned}
$$

The measurements are consistent with the tree level expectation $S_{J / \psi \pi^{0}}=-\sin 2 \beta$ and $C_{J / \psi \pi^{0}}=0$ but they have large statistical errors. More data is needed to draw a conclusion on a possible penguin contribution to the decay amplitude in this mode.

\subsection{2 $B^{0} \rightarrow D^{* \pm} D^{\mp}$}

The $B$ decays to the final states $D^{* \pm} D^{\mp}$ proceed dominantly through a $b \rightarrow c \bar{c} d$ tree amplitude. Deviations between the $C P$ asymmetries in $B^{0} \rightarrow D^{* \pm} D^{\mp}$ and in $B^{0} \rightarrow$ $J / \psi K_{S}^{0}$ due to contributions from penguin amplitudes from Standard Model processes are expected to be relatively small, of order $\Delta \beta=0.1 .{ }^{17,18}$ A number of processes from non-Standard Model physics, which can provide additional sources of $C P$ violation, can greatly increase this contribution, up to $\Delta \beta \approx 0.6$ in some models. ${ }^{17}$

The final states $D^{* \pm} D^{\mp}$ are not $C P$ eigenstates and four flavor-charge combinations must be considered $\left(B^{0}\left(\bar{B}^{0}\right) \rightarrow D^{* \pm} D^{\mp}\right)$. In the case of equal amplitudes for $B \rightarrow$ $D^{*-} D^{+}$and $B \rightarrow D^{*+} D^{-}$, one expects at tree level $C_{-+}=C_{+-}=0$ and $S_{-+}=$ $S_{+-}=-\sin 2 \beta$ for the corresponding $C P$ asymmetries.

Using a sample of 88 million $B^{0} \bar{B}^{0}$ decays $B A B A R$ has measured the time-dependent $C P$-asymmetries in $B^{0} \rightarrow D^{* \pm} D^{\mp}$ to be ${ }^{19}$

$$
\begin{aligned}
& S_{-+}=-0.24 \pm 0.69 \pm 0.12, \quad S_{+-}=-0.82 \pm 0.75 \pm 0.14, \\
& C_{-+}=-0.22 \pm 0.37 \pm 0.10, \quad C_{+-}=-0.47 \pm 0.40 \pm 0.12,
\end{aligned}
$$


consistent with the tree level expectation. In addition, $B A B A R$ has measured the timeindependent decay rate asymmetry between $B^{0} / \bar{B}^{0} \rightarrow D^{*+} D^{-}$and $B^{0} / \bar{B}^{0} \rightarrow D^{*-} D^{+}$ to be $-0.03 \pm 0.11 \pm 0.05$. More data is required to determine a possible penguin contribution to the decay amplitude.

\subsection{3 $\quad B^{0} \rightarrow D^{*+} D^{*-}$}

The final state $D^{*+} D^{*-}$ has the same quarks combinations bound to mesons as $D^{* \pm} D^{\mp}$ and, thus, suffers similarly from the simultaneous presence of tree and penguin amplitudes.

In the absence of penguin amplitudes the $C P$ asymmetry in $B^{0} \rightarrow D^{*+} D^{*-}$ is related to $\sin 2 \beta{ }^{20,21}$ Penguin-induced corrections are expected to be small in models based on the factorization approximation and heavy-quark symmetry; an effect of about $2 \%$ has been predicted. ${ }^{22}$ A comparison of measurements of $\sin 2 \beta$ from $b \rightarrow c \bar{c} s$ modes such as $B^{0} \rightarrow J / \psi K_{S}^{0}$ with that obtained in $B^{0} \rightarrow D^{*+} D^{*-}$ is an important test of these models.

The $B^{0} \rightarrow D^{*+} D^{*-}$ mode is a decay of a pseudo-scalar meson to a vector-vector final state, with contributions from three partial waves with different $C P$ parities: even for the $S$ - and $D$-waves, odd for the $P$-wave. The $C P$-odd contribution is predicted to be about $6 \%$. $^{23,24}$

$B A B A R^{25}$ uses a sample of 88 million $B^{0} \bar{B}^{0}$ decays to determine the $C P$-odd fraction in $B^{0} \rightarrow D^{*+} D^{*-}$ decays based on a one-dimensional time-integrated angular analysis to be $R_{\perp}=0.063 \pm 0.055 \pm 0.009$. Thus the final state is about $94 \% C P$-even. The $C P$-violating parameters for the $C P$-even component are obtained from a combined analysis of the time dependence of flavor-tagged decays and the one-dimensional angular distribution of the decay products:

$$
\left|\lambda_{+}\right|=0.75 \pm 0.19 \pm 0.02, \quad \mathcal{I} m \lambda_{+}=0.05 \pm 0.29 \pm 0.10 .
$$

The $\Delta t$ distributions of the selected events are shown in Fig. 7 together with the result from the likelihood fit. The measurements deviate by about $2.5 \sigma$ from the treelevel expectation $\left|\lambda_{+}\right|=1$ and $\mathcal{I} m \lambda_{+}=-\sin 2 \beta$. More data is needed to establish this deviation as a possible contribution from penguin processes. 


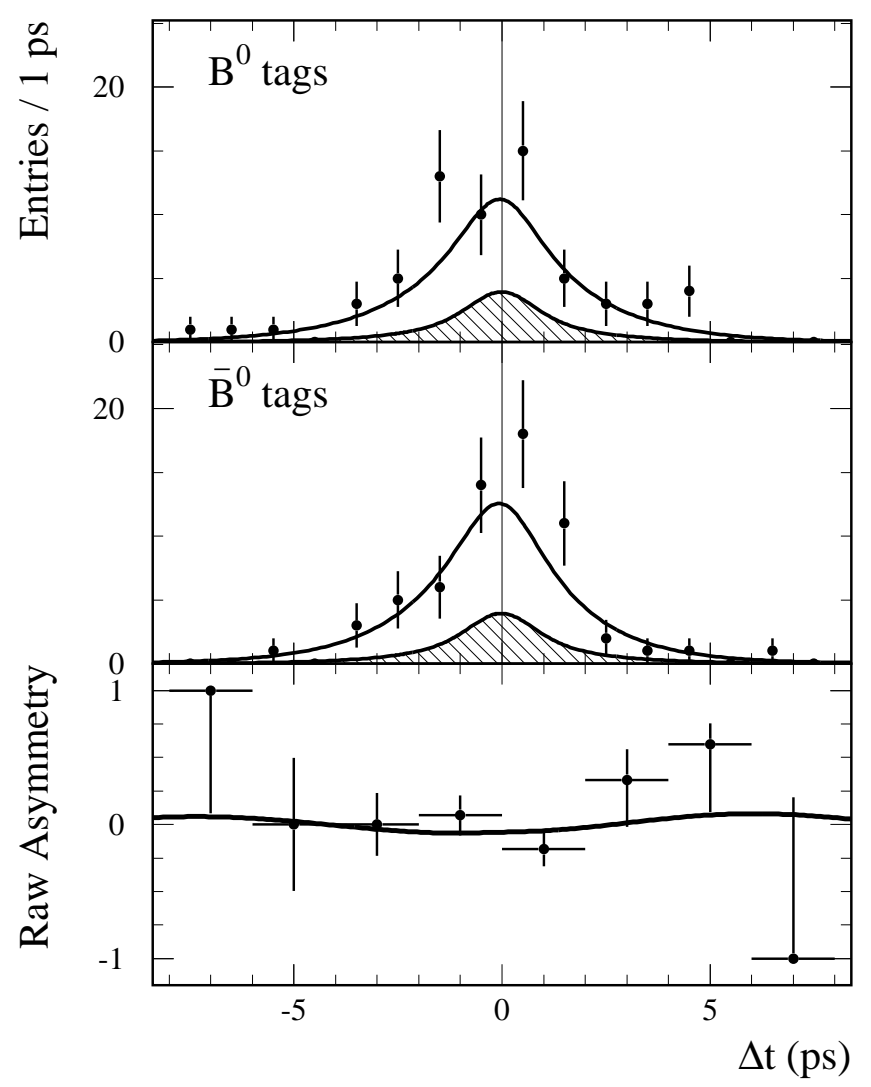

Fig. 7. $\Delta t$ distributions for the selected $B^{0}$-tagged (top) and $\bar{B}^{0}$-tagged (middle) $B \rightarrow D^{*+} D^{*-}$ candidates. Raw flavor asymmetry between $B^{0}$-tagged and $\bar{B}^{0}$-tagged candidates as a function of $\Delta t$ (bottom).

\subsection{Penguin-Dominated Modes}

The amplitudes for the decays $b \rightarrow s(\bar{s} s)$ and $b \rightarrow s(\bar{d} d)$ are dominated by the $b \rightarrow$ $s$ gluonic penguin diagram (see Fig. 8) where the top quark provides the dominant contribution to the quark loop. The leading penguin amplitude has the same weak phase as the $b \rightarrow c \bar{c} s$ tree amplitude. Thus, in the Standard Model the decays to the $\phi K_{S}^{0}$, $\eta^{\prime} K_{S}^{0}$ final states that proceed dominantly via a gluonic penguin diagram are expected to have the same $C P$-asymmetry as $B^{0} \rightarrow J / \psi K_{S}^{0}$.

There is significant interest in these decays. They offer a natural place for sizeable contributions from new physics processes due to their relatively small branching fractions and the possibility that the (virtual) Standard Model particles in the loop (top and $W$ ) can be replaced by new particles (e.g. SUSY). 

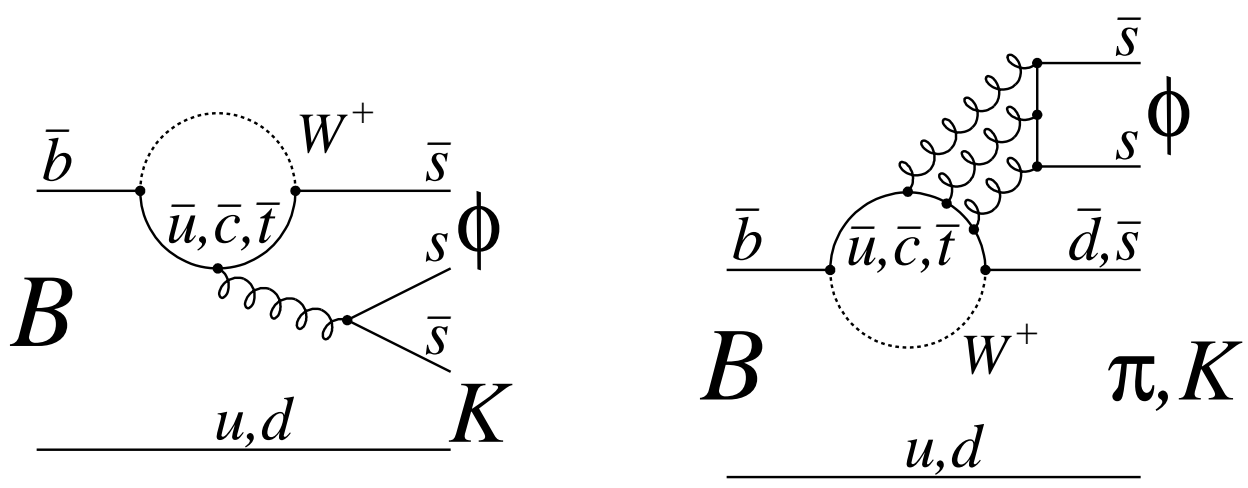

Fig. 8. Diagrams for the $b \rightarrow s \bar{s} s$ gluonic-penguin amplitude leading to the decay $B^{0} \rightarrow \phi K_{S}^{0}$.

\subsection{1 $\quad B^{0} \rightarrow \phi K_{S}^{0}$}

In addition to the leading penguin amplitude there are small contributions from transitions with different weak phases that contribute to $B^{0} \rightarrow \phi K_{S}^{0}$. Their magnitude can be limited from measurements of related decay modes such as $B^{+} \rightarrow \phi \pi^{+}, K^{* 0} K^{+}$. The deviation of $S_{\phi K_{S}^{0}}$ from $\sin 2 \beta$ is expected to be less than $5 \% .^{26} B A B A R^{27}$ and Belle $^{28}$ have measured the $C P$-asymmetries in $B^{0} \rightarrow \phi K_{S}^{0}$ with $81 \mathrm{fb}^{-1}$ and $78 \mathrm{fb}^{-1}$, respectively, to be

$$
\begin{array}{ll}
S_{\phi K_{S}^{0}}=-0.18 \pm 0.51 \pm 0.07 & C_{\phi K_{S}^{0}}=-0.80 \pm 0.38 \pm 0.12 \quad \text { (BABAR), } \\
S_{\phi K_{S}^{0}}=-0.73 \pm 0.64 \pm 0.22 & C_{\phi K_{S}^{0}}=0.56 \pm 0.41 \pm 0.16 \quad \text { (Belle) } .
\end{array}
$$

The $\Delta t$ distributions are shown in Figs. 9 (BABAR) and 10 (Belle). The average of $S_{\phi K_{S}^{0}}$ deviates by about $2 \sigma$ from the sine coefficient in $B^{0} \rightarrow J / \psi K_{S}^{0}$. Belle has also measured the time-dependent $C P$ asymmetry for non-resonant $B^{0} \rightarrow K^{+} K^{-} K_{S}^{0}$ which has contributions from $C P$-even and $C P$-odd partial waves. They measure the $C P$-even fraction to be $97 \%$ and the $C P$-asymmetries

$$
S_{K K K_{S}^{0}}=-0.49 \pm 0.43 \pm 0.11_{-0.00}^{+0.33} \quad C_{K K K_{S}^{0}}=-0.40 \pm 0.33 \pm 0.10_{-0.26}^{+0.00}
$$

consistent with the Standard Model expectation of $S_{K K K_{S}^{0}}=-\sin 2 \beta$ and $C_{K K K_{S}^{0}}=$ 0 assuming a pure $C P$-even final state. 

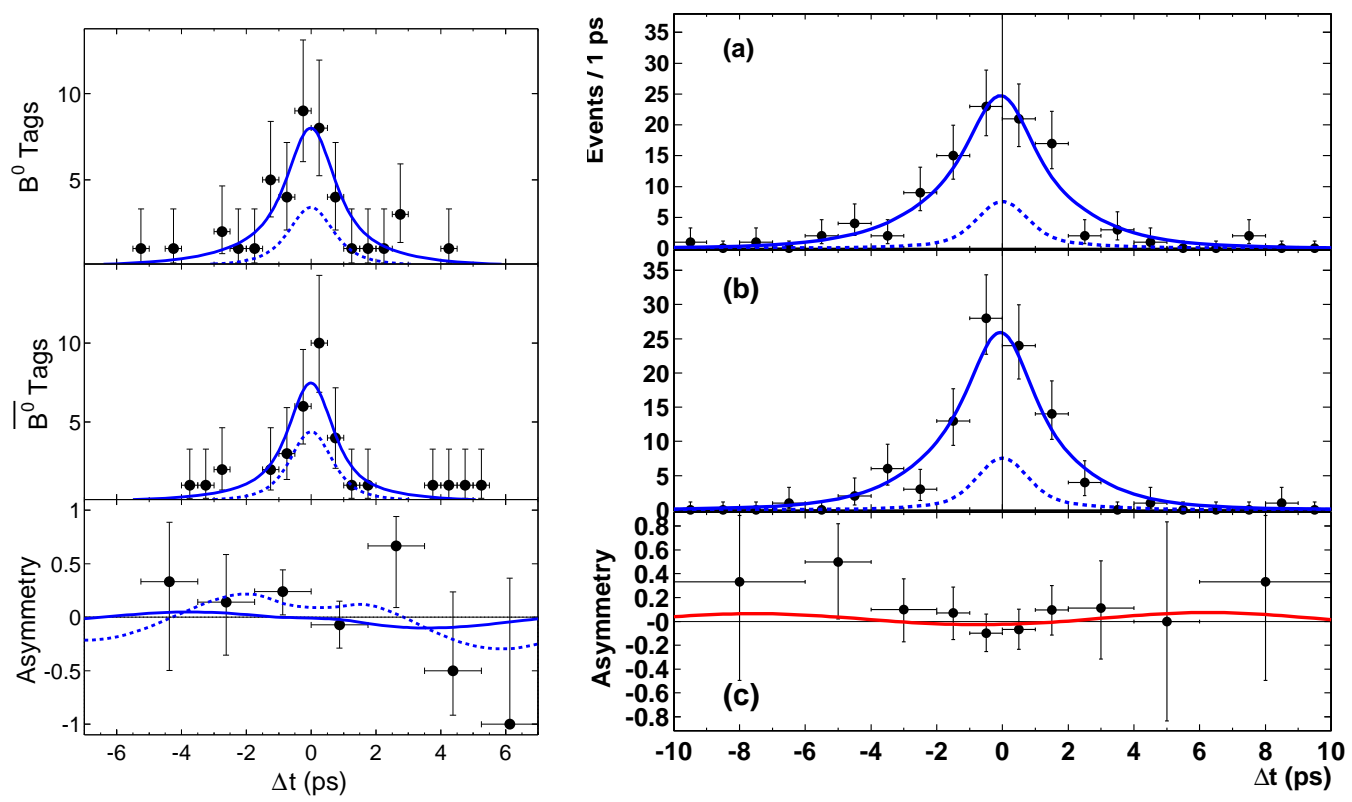

Fig. 9. Distributions of $\Delta t$ for $B^{0}$-tagged events (top) and $\bar{B}^{0}$-tagged events (middle) and raw flavor asymmetry (bottom) for the selected $B \rightarrow \phi K_{S}^{0}$ (left) and $B \rightarrow \eta^{\prime} K_{S}^{0}$ (right) events from $B A B A R$.

\subsection{2 $\quad B^{0} \rightarrow \eta^{\prime} K_{S}^{0}$}

In the decay $B^{0} \rightarrow \eta^{\prime} K_{S}^{0}$ the internal gluon can convert to either an $s \bar{s}$ or $d \bar{d}$ pair. The relatively large branching fraction of $6 \times 10^{-5}$ could be the result of constructive interference of these amplitudes. In addition there is a contribution from a CKM-suppressed tree-level $b \rightarrow u$ transition. Estimates of the relative tree and penguin amplitudes expect a deviation of $S_{\eta^{\prime} K_{S}^{0}}$ from $\sin 2 \beta$ to be less than about $5 \%{ }^{29} B A B A R^{30}$ and Belle ${ }^{31}$ have measured the time-dependent $C P$ asymmetries in $B^{0} \rightarrow \eta^{\prime} K_{S}^{0}$ decays with $82 \mathrm{fb}^{-1}$ and $78 \mathrm{fb}^{-1}$, respectively:

$$
\begin{aligned}
& S_{\eta^{\prime} K_{S}^{0}}=0.02 \pm 0.34 \pm 0.03 \quad C_{\eta^{\prime} K_{S}^{0}}=0.10 \pm 0.22 \pm 0.04 \quad \text { (BABAR), } \\
& S_{\eta^{\prime} K_{S}^{0}}=0.71 \pm 0.37 \pm 0.06 \quad C_{\eta^{\prime} K_{S}^{0}}=-0.26 \pm 0.22 \pm 0.04 \quad \text { (Belle). }
\end{aligned}
$$

The $\Delta t$ distributions of samples enriched in $B^{0} \rightarrow \eta^{\prime} K_{S}^{0}$ events are shown in Figs. 9 (BABAR) and 10 (Belle). The measured coefficients $S_{\eta^{\prime} K_{S}^{0}}$ and $C_{\eta^{\prime} K_{S}^{0}}$ are consistent with $\sin 2 \beta$ and zero, respectively. 

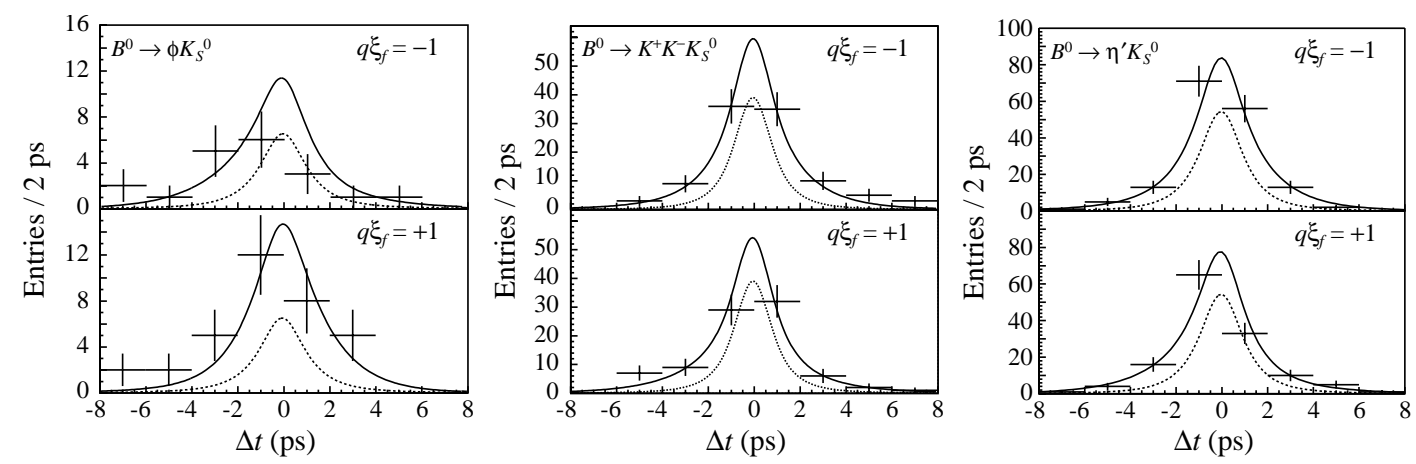

Fig. 10. Distributions of $\Delta t$ for the selected $B \rightarrow \phi K_{S}^{0}$ (left), $B \rightarrow K^{+} K^{-} K_{S}^{0}$ (middle), and $B \rightarrow \eta^{\prime} K_{S}^{0}$ (right) candidates from Belle.

\subsubsection{Charmonium Modes versus Penguin-dominated Modes}

The measurements of $\sin 2 \beta$ with charmonium modes which proceed through a $b \rightarrow c \bar{c} s$ tree amplitude and the penguin-dominated modes which proceed dominantly through a $b \rightarrow s$ penguin amplitude are summarized in Fig. $11 .{ }^{13}$ The shaded bands show separately the averages of the measurements for $B$ decays to charmonium and for $B$ decays to penguin-dominated modes. The two bands differ by about 2.6 standard deviations. A future confirmation of the separation of the two bands would challenge the Standard Model and could provide the first evidence for new physics contributions to $C P$ violation.

\section{Measurement sensitive to $\sin 2 \alpha$}

Decay modes to hadronic final states with a dominant contribution from $b \rightarrow u \bar{u} d$ are sensitive to the CKM angle $\alpha$. Recent measurements in the decay modes $B^{0} \rightarrow \pi^{+} \pi^{-}$ and $B^{0} \rightarrow \rho^{ \pm} \pi^{\mp}$ are presented.

\section{1 $\quad B^{0} \rightarrow \pi^{+} \pi^{-}$}

The decay $B^{0} \rightarrow \pi^{+} \pi^{-}$is expected to proceed dominantly through a $b \rightarrow u W^{-}$tree amplitude. The weak phase difference $2 \gamma$ between the tree amplitudes for a $B^{0}$ and a $\bar{B}^{0}$ to the $\pi^{+} \pi^{-}$final state adds to the weak phase difference $2 \beta$ from $B^{0} \bar{B}^{0}$ mixing to a combined weak phase difference of $2 \alpha$, where $\alpha=\arg \left[-V_{t d} V_{t b}^{*} / V_{u d} V_{u b}^{*}\right]$. Thus, if the decay proceeds purely through the tree amplitude, the complex parameter $\lambda_{\pi^{+} \pi^{-}}$is 


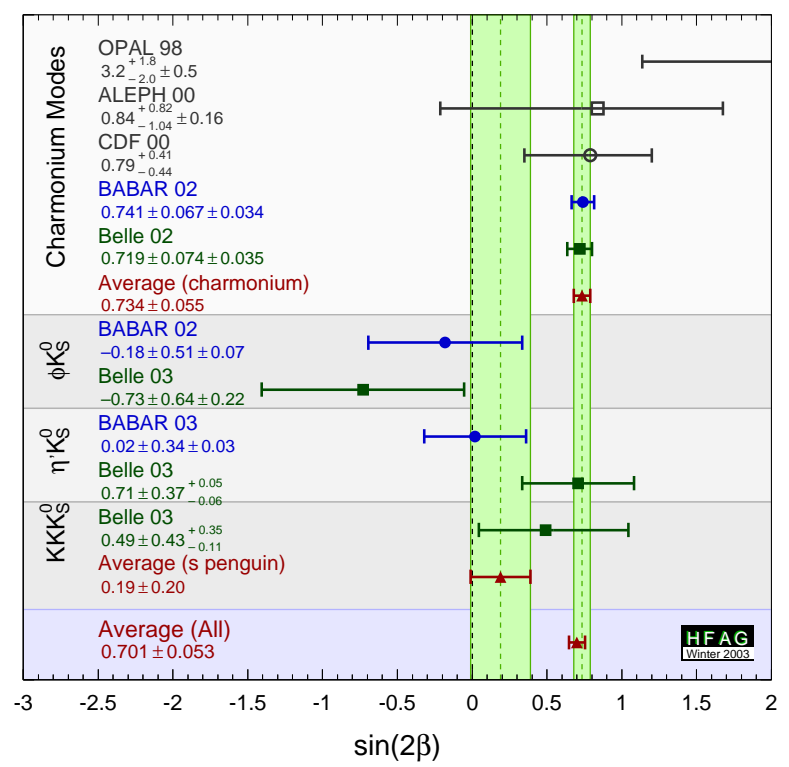

Fig. 11. Measurements of $\sin 2 \beta$ with charmonium modes and penguin-dominated modes and their world averages.

directly related to CKM matrix elements and $\left|\lambda_{\pi \pi}\right|=1$ and $\mathcal{I} m \lambda_{\pi^{+} \pi^{-}}=\sin 2 \alpha$.

However, possible significant contributions from a $b \rightarrow d g$ gluonic penguin amplitude at the same order in $\lambda$ as the tree amplitude with different weak and strong phases complicate the situation. Theoretical estimates suggest that the contribution from the gluonic penguin amplitude can be significant. ${ }^{32}$ Therefore, the time-dependent $C P$ violating asymmetry in the decay $B^{0} \rightarrow \pi^{+} \pi^{-}$arises from interference between mixing and decay amplitudes, and interference between the $b \rightarrow u W^{-}$and $b \rightarrow d g$ decay amplitudes.

In general, $S_{\pi \pi}$ can be expressed as $\sqrt{1-C_{\pi^{+} \pi^{-}}^{2}} \sin 2 \alpha_{\text {eff }}$ where $\alpha_{\text {eff }}=\alpha+\Delta \alpha$ depends on the magnitudes and relative weak and strong phases of the tree and penguin amplitudes. Consequently, $S_{\pi \pi}$ may differ from $\sin 2 \alpha$ and direct $C P$ violation $\left(C_{\pi^{+} \pi^{-}} \neq\right.$ $0)$ can be present.

$B A B A R$ and Belle reconstruct $B^{0} \rightarrow \pi^{+} \pi^{-}$events in samples of $88 \times 10^{6}$ and $85 \times 10^{6}$ $B \bar{B}$ pairs, respectively. In Fig. 12 the distributions of $\triangle E$ for samples enriched in $B^{0} \rightarrow \pi^{+} \pi^{-}$events from BABAR and Belle are shown.

The $C P$-asymmetries are determined with an unbinned likelihood fit to the $\Delta t$ distributions of the selected events. Belle imposes relatively tight requirements for the selected events on $m_{\mathrm{ES}}, \triangle E$, and event topology variables. BABAR employs looser 

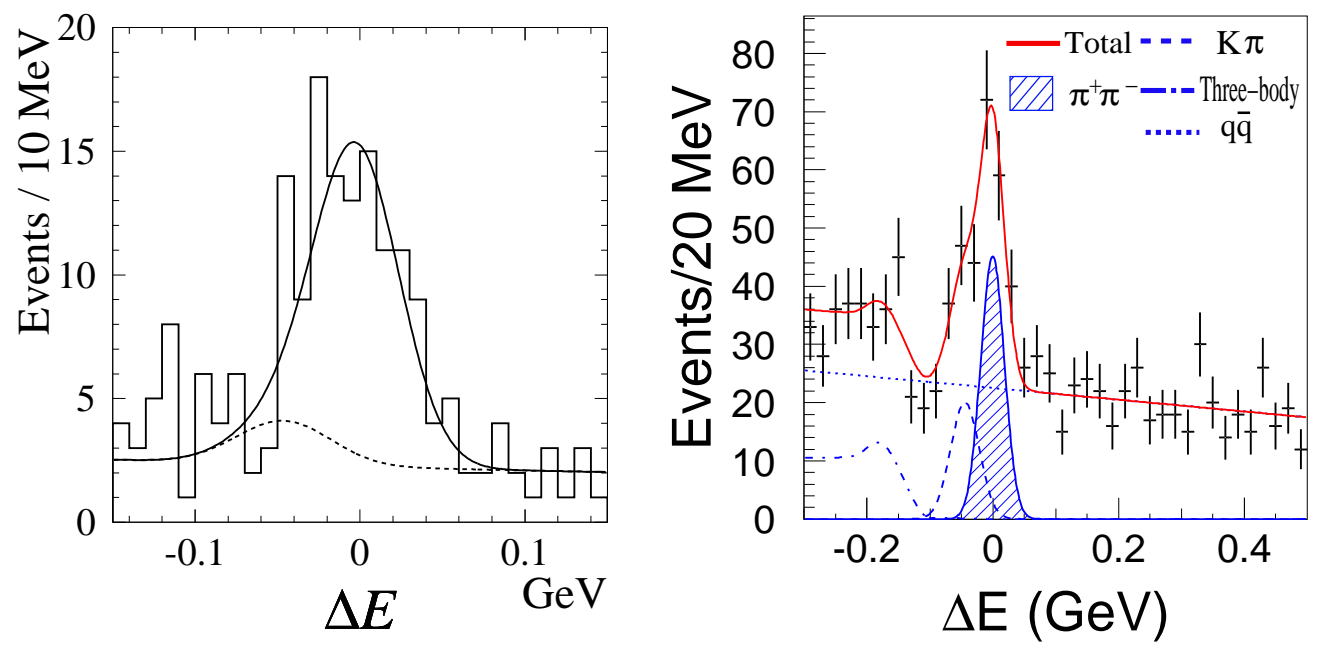

Fig. 12. Distributions of $\triangle E$ for samples enriched in $B^{0} \rightarrow \pi^{+} \pi^{-}$events from $B A B A R$ and Belle. BABAR (left): The solid curve represents the projection of the maximum likelihood fit result, while the dashed curve represents $q \bar{q}$ and $K \pi$ cross-feed background. Belle (right): The solid curve represents the projection of the maximum likelihood fit result. The dotted curve represents continuum background, the dashed curve represents $K \pi$ cross-feed background and the dot-dashed curve represents charmless threebody $B$ decay background. The hashed area represents the $B \rightarrow \pi^{+} \pi^{-}$signal candidates. 
requirements in the event selection and uses in $m_{\mathrm{ES}}, \Delta E$, and a Fisher discriminant that describes the energy flow in the event in the unbinned likelihood fit to discriminate between signal and background. The $\Delta t$ distributions of samples enriched in $B^{0} \rightarrow \pi^{+} \pi^{-}$ events are shown in Fig. 13.
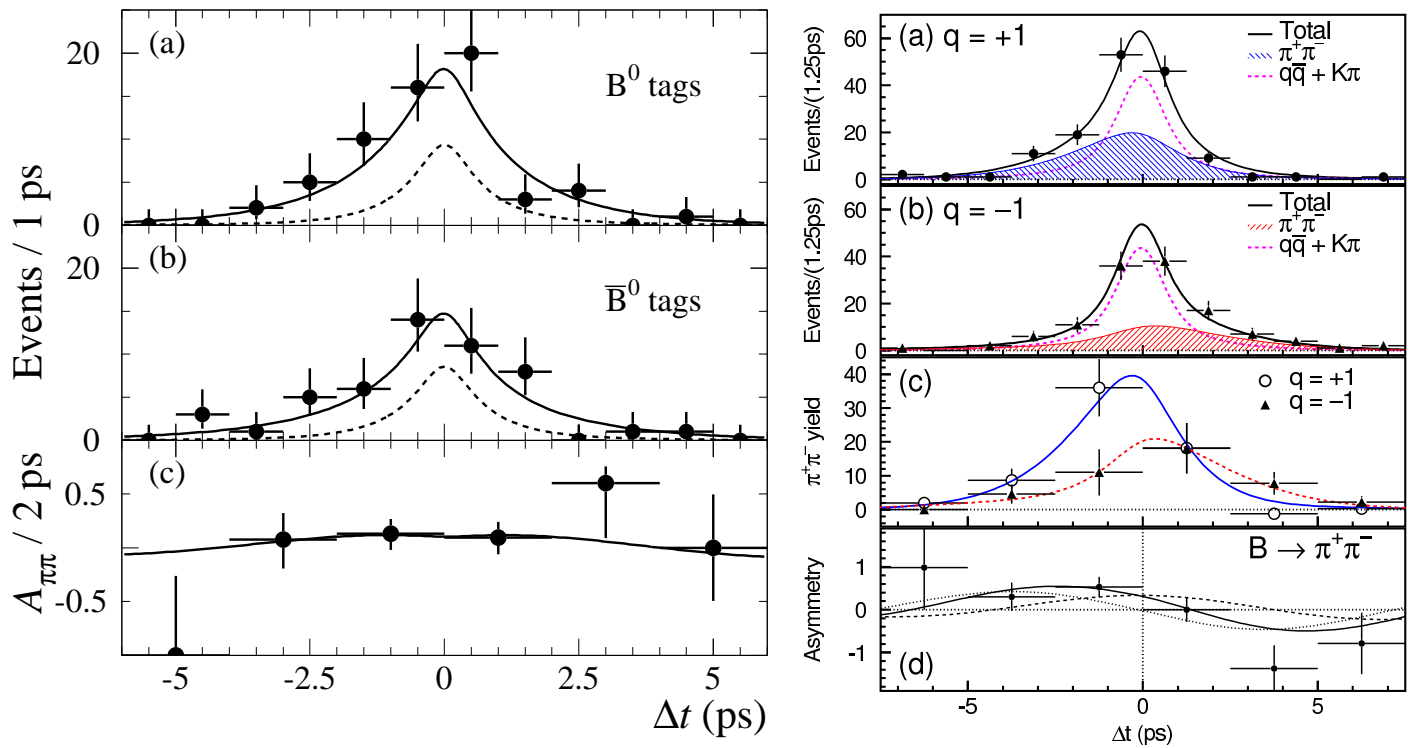

Fig. 13. Distributions of $\Delta t$ and raw flavor asymmetries for samples enriched in $B^{0} \rightarrow$ $\pi^{+} \pi^{-}$candidates from $B A B A R$ and Belle. BABAR (left): Distributions of $\Delta t$ for events with (a) $B_{\text {tag }}=B^{0}$ or (b) $B_{\text {tag }}=\bar{B}^{0}$ and (c) raw asymmetry between $B^{0}$ and $\bar{B}^{0}$-tagged events. Solid curves represent projections of the likelihood fit, dashed curves represent the sum of $q \bar{q}$ and $K \pi$ background. Belle (right): Distributions of $\Delta t$ for events with (a) $B_{\text {tag }}=B^{0}$ or (b) $B_{\text {tag }}=\bar{B}^{0}$ and (c) background subtracted events. The $C P$ asymmetry after background subtraction between $B^{0}$ and $\bar{B}^{0}$-tagged events is shown in (d). Solid curves represent projections of the likelihood fit, dashed curves represent the sum of $q \bar{q}$ and $K \pi$ background. The shaded region represents the contribution from signal $\pi \pi$. In Fig. (d), the dashed and dotted curves represent the contributions from the cosine and sine terms, respectively.

$B A B A R^{33}$ and Belle ${ }^{34}$ measure the $C P$-asymmetries in $B^{0} \rightarrow \pi^{+} \pi^{-}$events to be

$$
\begin{array}{lll}
S_{\pi \pi}=-0.30 \pm 0.25 \pm 0.04 & C_{\pi \pi}=-0.02 \pm 0.34 \pm 0.05 & \text { (BABAR), } \\
S_{\pi \pi}=-1.23 \pm 0.41 \pm 0.08 & C_{\pi \pi}=-0.77 \pm 0.27 \pm 0.08 & \text { (Belle). }
\end{array}
$$

The average values of $S_{\pi \pi}$ and $C_{\pi \pi}$ from combining the results from $B A B A R$ and Belle are 


$$
S_{\pi \pi}=-0.47 \pm 0.26 \text { and } C_{\pi \pi}=-0.49 \pm 0.19
$$

The confidence intervals for the $B A B A R$ and Belle measurements in the $-C_{\pi \pi}$ versus $S_{\pi \pi}$ plane are shown in Fig. $14 .^{35}$ The BABAR and Belle measurements differ by $2.2 \sigma$. More data is needed to resolve the discrepancy in the future.

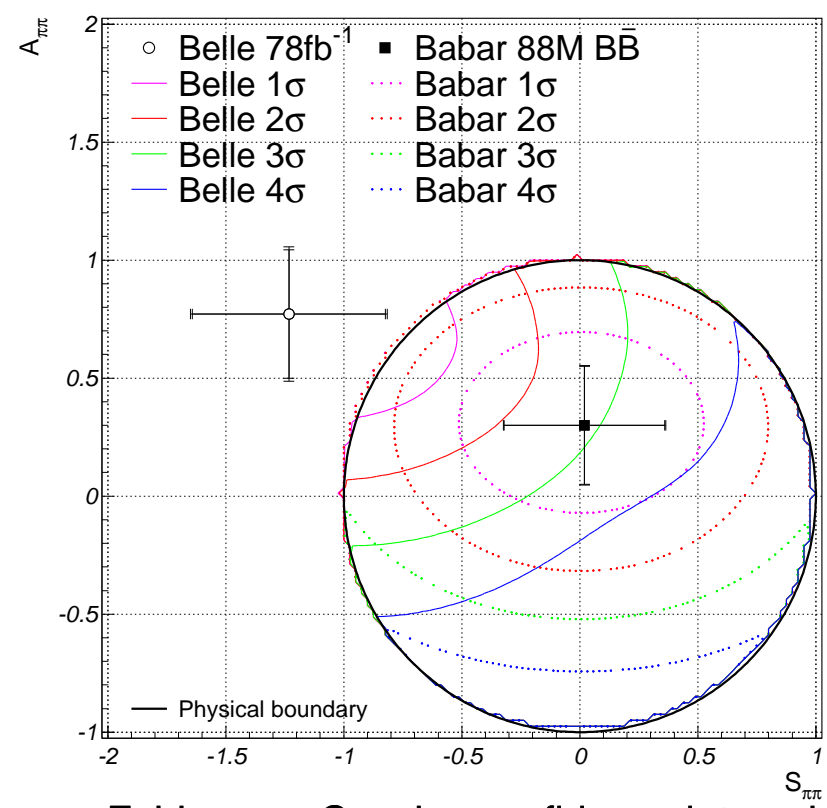

Feldman \& Cousins confidence interval

Fig. 14. Confidence regions for $-C_{\pi \pi}\left(=A_{\pi \pi}\right)$ versus $S_{\pi \pi}$ for the $B A B A R$ and Belle measurements.

The CKM angle $\alpha$ can be determined without large theoretical uncertainties with an isospin analysis ${ }^{36}$ if in addition to the time-dependent $C P$-asymmetries in $B \rightarrow \pi^{+} \pi^{-}$ also the decay rates for $B^{ \pm} \rightarrow \pi^{ \pm} \pi^{0}, B^{0} \rightarrow \pi^{0} \pi^{0}$, and $\bar{B}^{0} \rightarrow \pi^{0} \pi^{0}$ are measured. Based on a sample of $124 \times 10^{6} B \bar{B}$ pairs $B A B A R^{37}$ has measured the branching fraction for $B \rightarrow \pi^{0} \pi^{0}$ averaged over $B^{0}$ and $\bar{B}^{0}$ to be $(2.1 \pm 0.6 \pm 0.3) \times 10^{-6}$. The signal is observed at a significance of $4.2 \sigma$ including systematic uncertainties. In $152 \times 10^{6} \mathrm{~B} \bar{B}$ pairs Belle ${ }^{38}$ observes evidence for this decay at the $3.4 \sigma$ level and measures a branching fraction of $(1.7 \pm 0.6 \pm 0.2) \times 10^{-6}$ in good agreement with the BABAR measurement.

Using isospin relations a small value of the $B \rightarrow \pi^{0} \pi^{0}$ branching fraction could give an upper bound on $\left|\alpha_{\text {eff }}-\alpha\right| .^{39,40}$ However, the measured central values of the branching fractions for $B \rightarrow \pi^{0} \pi^{0}, B \rightarrow \pi^{+} \pi^{-}$, and $B^{+} \rightarrow \pi^{+} \pi^{0}$ give only a very weak constraint of $\left|\alpha_{\text {eff }}-\alpha\right|>54^{\circ}$. Without additional input from theoretical models 
about $10 \mathrm{ab}^{-1}$ will be needed to discriminate between the large and small $\left|\alpha_{\text {eff }}-\alpha\right|$ solutions. ${ }^{41}$

\section{2 $B^{0} \rightarrow \rho^{ \pm} \pi^{\mp}$}

Similar to the decay $B^{0} \rightarrow \pi^{+} \pi^{-}$, the decay $B^{0} \rightarrow \rho^{ \pm} \pi^{\mp}$ proceeds dominantly via a $b \rightarrow u$ tree diagram or a $b \rightarrow d$ penguin diagram. However, unlike $B^{0} \rightarrow \pi^{+} \pi^{-}, B^{0} \rightarrow$ $\rho^{ \pm} \pi^{\mp}$ is not a $C P$ eigenstate and four flavor-charge combinations must be considered $\left(B^{0}\left(\bar{B}^{0}\right) \rightarrow \rho^{ \pm} \pi^{\mp}\right)$. This requires more $C P$ parameters to describe the time-dependence of the decay, but the mode $B^{0} \rightarrow \rho^{ \pm} \pi^{\mp}$ benefits from an almost five times larger branching fraction.

In principle, the CKM angle $\alpha$ can be measured in the presence of penguin contributions without large theoretical uncertainties by using a Dalitz plot analysis of the $\pi^{+} \pi^{-} \pi^{0}$ final state. However, due to low $\pi^{0}$ reconstruction efficiency in certain areas of the Dalitz plot and large backgrounds from mis-reconstructed signal events and other $B$ decays such an analysis requires large statistics.

$B A B A R^{42}$ has performed an analysis based on a quasi-two-body approach by restricting the time-dependent fit to events in the two regions of the Dalitz plot that are dominated by $B \rightarrow \rho^{ \pm} \pi^{\mp}$. The time-dependent decay rates for $B^{0}\left(\bar{B}^{0}\right) \rightarrow \rho^{ \pm} \pi^{\mp}$ are given by

$$
\begin{aligned}
f_{q}^{\rho^{ \pm} \pi^{\mp}}(\Delta t) & =\left(1 \pm A_{C P}^{\rho \pi}\right) \frac{e^{-|\Delta t| / \tau}}{4 \tau} \\
& \times\left[1+q\left(S_{\rho \pi} \pm \Delta S_{\rho \pi}\right) \sin \left(\Delta m_{B} \Delta t\right)-q\left(C_{\rho \pi} \pm \Delta C_{\rho \pi}\right) \cos \left(\Delta m_{B} \Delta t\right)\right]
\end{aligned}
$$

where $q=+1(-1)$ when $B_{\operatorname{tag}}$ is a $B^{0}\left(\bar{B}^{0}\right)$. The time- and flavor-integrated charge asymmetries $A_{C P}^{\rho \pi}$ measure direct $C P$-violation and the parameters $S_{\rho \pi}$ and $C_{\rho \pi}$ parameterize mixing-induced $C P$-violation related to the CKM angle $\alpha$ and flavor dependent direct $C P$-violation, respectively. The parameter $\Delta C_{\rho \pi}$ describes the asymmetry between the decay rates $\Gamma\left(B^{0} \rightarrow \rho^{+} \pi^{-}\right)+\Gamma\left(\bar{B}^{0} \rightarrow \rho^{-} \pi^{+}\right)$and $\Gamma\left(B^{0} \rightarrow \rho^{-} \pi^{+}\right)+\Gamma\left(\bar{B}^{0} \rightarrow\right.$ $\left.\rho^{+} \pi^{-}\right)$. The parameter $\Delta S_{\rho \pi}$ is related to the strong phase difference between the amplitudes contributing to the $B \rightarrow \rho^{ \pm} \pi^{\mp}$ decays. The parameters are related via $S_{\rho \pi} \pm \Delta S_{\rho \pi}=\sqrt{1-\left(C_{\rho \pi} \pm \Delta C_{\rho \pi}\right)^{2}} \sin \left(2 \alpha_{\text {eff }}^{ \pm} \pm \delta\right)$ where $2 \alpha_{\text {eff }}^{ \pm}=\arg \left[(q / p) \bar{A}_{\rho \pi}^{ \pm} / A_{\rho \pi}^{\mp}\right]$, $\delta=\arg \left[A_{\rho \pi}^{-} / A_{\rho \pi}^{+}\right]$, and $A_{\rho \pi}^{+}\left(\bar{A}_{\rho \pi}^{+}\right)$and $A_{\rho \pi}^{-}\left(\bar{A}_{\rho \pi}^{-}\right)$are the transition amplitudes for the processes $B^{0}\left(\bar{B}^{0}\right) \rightarrow \rho^{+} \pi^{-}$and $B^{0}\left(\bar{B}^{0}\right) \rightarrow \rho^{-} \pi^{+}$, respectively. The angles $\alpha_{\text {eff }}^{ \pm}$are expected to be equal to $\alpha$ in the absence of contributions from penguin amplitudes. 


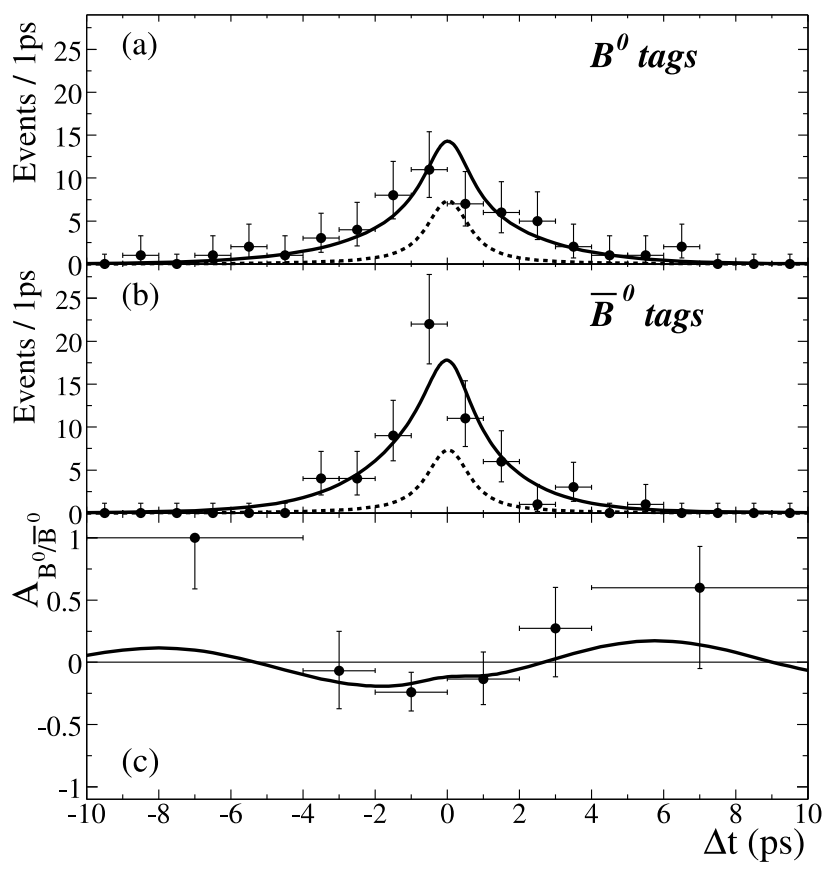

Fig. 15. Distributions of $\Delta t$ for kaon and lepton tagged $B \rightarrow \rho^{ \pm} \pi^{\mp}$ candidate events where $B_{\text {tag }}$ is a (a) $B^{0}$, (b) $\bar{B}^{0}$, and (c) raw flavor asymmetry between $B^{0}$ and $\bar{B}^{0}$ tagged events. The solid line presents the likelihood fit result and the dashed line presents the background contribution.

With a sample of 89 million $B \bar{B}$ pairs, BABAR finds $428 \pm 34 B \rightarrow \rho^{ \pm} \pi^{\mp}$ events. The $\Delta t$ distributions for events enriched in $B \rightarrow \rho^{ \pm} \pi^{\mp}$ decays are shown in Fig. 15 . The $C P$-asymmetries are determined from an unbinned likelihood fit to be

$$
\begin{array}{rlrl}
A_{C P}^{\rho \pi} & =-0.18 \pm 0.08 \pm 0.03, & \Delta S_{C P}^{\rho \pi}=0.15 \pm 0.25 \pm 0.03, \\
S_{C P}^{\rho \pi}=0.19 \pm 0.24 \pm 0.03, & \Delta C_{C P}^{\rho \pi}=0.28 \pm 0.19 \pm 0.04, \\
C_{C P}^{\rho \pi}=0.36 \pm 0.18 \pm 0.04 . &
\end{array}
$$

The measurement of direct $C P$-asymmetry can be expressed as

$$
\begin{aligned}
& A_{+-}=\frac{N\left(\bar{B}^{0} \rightarrow \rho^{+} \pi^{-}\right)-N\left(B^{0} \rightarrow \rho^{-} \pi^{+}\right)}{N\left(\bar{B}^{0} \rightarrow \rho^{+} \pi^{-}\right)+N\left(B^{0} \rightarrow \rho^{-} \pi^{+}\right)}=\frac{A_{C P}^{\rho \pi}-C_{C P}^{\rho \pi}-A_{C P}^{\rho \pi} \Delta C_{C P}^{\rho \pi}}{1-\Delta C_{C P}-A_{C P}^{\rho \pi} C_{C P}^{\rho \pi}} \\
& A_{-+}=\frac{N\left(\bar{B}^{0} \rightarrow \rho^{-} \pi^{+}\right)-N\left(B^{0} \rightarrow \rho^{+} \pi^{-}\right)}{N\left(\bar{B}^{0} \rightarrow \rho^{-} \pi^{+}\right)+N\left(B^{0} \rightarrow \rho^{+} \pi^{-}\right)}=\frac{A_{C P}^{\rho \pi}+C_{C P}^{\rho \pi}+A_{C P}^{\rho \pi} \Delta C_{C P}^{\rho \pi}}{1+\Delta C_{C P}+A_{C P}^{\rho \pi} C_{C P}^{\rho \pi}}
\end{aligned}
$$

which $B A B A R$ measures to be

$$
\begin{aligned}
& A_{+-}=-0.62_{-0.28}^{+0.24} \pm 0.06 \\
& A_{-+}=-0.11_{-0.17}^{+0.16} \pm 0.04
\end{aligned}
$$


The value for $A_{+-}$yields a hint of a deviation from no direct $C P$ violation of about $2 \sigma$. More data is required to measure direct or time-dependent $C P$-violation in the decay $B \rightarrow \rho^{ \pm} \pi^{\mp}$ at a significant level.

\section{Measurements sensitive to $\gamma$}

The angle $\gamma$ of the Unitarity Triangle is defined as $\gamma=\arg \left(-V_{u d} V_{u b}^{*} / V_{c d} V_{c b}^{*}\right)$. Thus, $B$ decay modes in which $b \rightarrow u$ tree amplitudes interfere with $b \rightarrow c$ tree amplitudes are sensitive to $\gamma$. Decays of the type $B \rightarrow D X$, where $X$ is one or more light mesons, which can be mediated by both of these amplitudes can be used to measure $\gamma$. These modes are theoretically clean, because the single charm quark in the final state ensures that there is no contribution from penguin amplitudes.

\section{$5.1 \quad B^{+} \rightarrow D K^{+}$}

The decay $B^{+} \rightarrow D K^{+}$can proceed via two different tree amplitudes. The amplitude for $\bar{b} \rightarrow \bar{c} u \bar{s}$ leads to the final state $\bar{D}^{0} K^{+}$and the amplitude for $\bar{b} \rightarrow \bar{u} c \bar{s}$ leads to the final state $D^{0} K^{+}$as shown Fig. 16. The two amplitudes differ by the weak phase $\gamma$ and can interfere, if $D^{0}$ and $\bar{D}^{0}$ decay to the same final state ${ }^{43}$ (e.g. a $C P$ eigenstate such as $\left.K^{+} K^{-}\right)$.
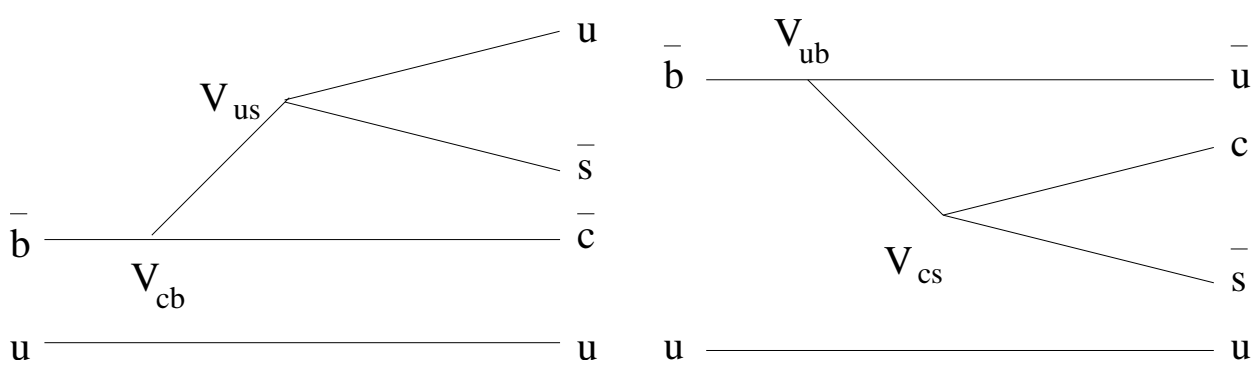

Fig. 16. Feynman-diagrams for the decay amplitudes of $B^{+} \rightarrow \bar{D}^{0} K^{+}$(left) and $B^{+} \rightarrow D^{0} K^{+}$(right).

In principle, the angle $\gamma$ can be extracted, if the rates of decay for $B^{+} \rightarrow D^{0} K^{+}$, $B^{-} \rightarrow \bar{D}^{0} K^{-}, B^{ \pm} \rightarrow D_{+} K^{ \pm}$, and $B^{+} \rightarrow \bar{D}^{0} K^{+}$are measured (the $C P$ eigenstates of the neutral $D$ meson are defined as $\left.D_{ \pm}=\left(D^{0} \pm \bar{D}^{0}\right) / \sqrt{2}\right)$. The relations between the involved amplitudes are sketched in Fig. 17. 


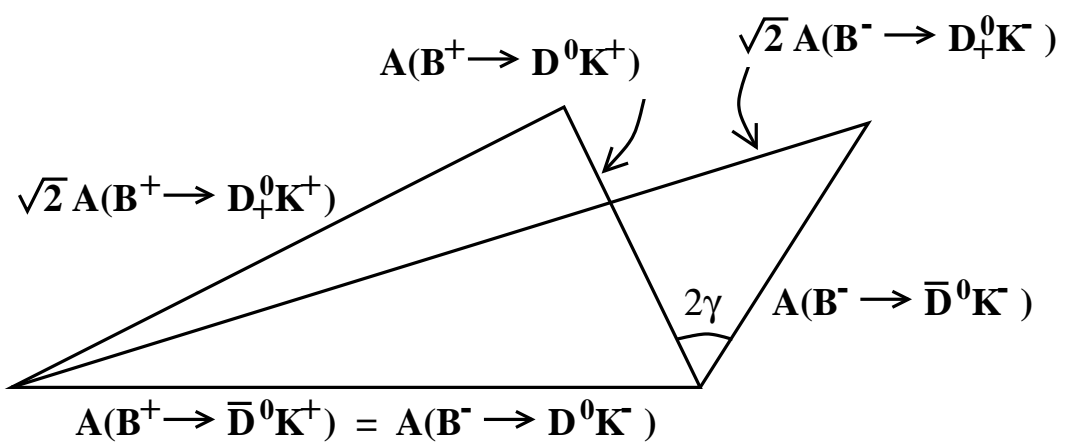

Fig. 17. Triangle relations between $B^{ \pm} \rightarrow D K^{ \pm}$amplitudes. $^{44}$

Unfortunately the branching fractions for $B^{+} \rightarrow D^{0} K^{+}$and $B^{-} \rightarrow \bar{D}^{0} K^{-}$cannot be determined with hadronic $D$ decays. The combined branching fraction for the decay under study $B^{+} \rightarrow D^{0} K^{+}$followed by a $D^{0}$ decay into a Cabibbo-favored final state (e.g. $D^{0} \rightarrow K^{-} \pi^{+}$) is expected to be of similar magnitude as the branching fraction for the decay $B^{+} \rightarrow \bar{D}^{0} K^{+}$followed by a doubly Cabibbo-suppressed $\bar{D}^{0}$ decay $\left(\bar{D}^{0} \rightarrow\right.$ $\left.K^{-} \pi^{+}\right)$. Still, from the measured $B^{ \pm} \rightarrow D K^{ \pm}$branching fractions $C P$-asymmetries $A_{ \pm}$and Cabibbo-suppression double ratios $R_{ \pm}$can be defined which are related to $\gamma$ and the ratio of the magnitudes $r$ and the strong phase difference $\delta$ between the $b \rightarrow u$ and $b \rightarrow c$ amplitudes:

$$
\begin{aligned}
A_{ \pm} & =\frac{\mathcal{B}\left(B^{-} \rightarrow D_{ \pm} K^{-}\right)-\mathcal{B}\left(B^{+} \rightarrow D_{ \pm} K^{+}\right)}{\mathcal{B}\left(B^{-} \rightarrow D_{ \pm} K^{-}\right)+\mathcal{B}\left(B^{+} \rightarrow D_{ \pm} K^{+}\right)}=\frac{ \pm 2 r \sin \delta \sin \gamma}{1+r^{2}+2 r \cos \delta \cos \gamma} \\
R_{ \pm} & =\frac{\mathcal{B}\left(D_{ \pm} K^{-}\right) / \mathcal{B}\left(D_{ \pm} \pi^{-}\right)}{\mathcal{B}\left(D^{0} K^{-}\right) / \mathcal{B}\left(D^{0} \pi^{-}\right)}=1+r^{2} \pm 2 r \cos \delta \cos \gamma
\end{aligned}
$$

$B A B A R^{45}$ and Belle ${ }^{46}$ have measured $A_{ \pm}$and $R_{ \pm}$in $B^{+} \rightarrow D K^{+}$decays. BABAR reconstructs the $D$ meson in the $C P$-even final state $K^{+} K^{-}$in $75 \mathrm{fb}^{-1}$ of data. Belle reconstructs the $D$ in the $C P$-even final states $K^{+} K^{-}$and $\pi^{+} \pi^{-}$and the $C P$-odd final states $K_{S}^{0} \pi^{0}, K_{S}^{0} \phi, K_{S}^{0} \omega, K_{S}^{0} \eta$ and $K_{S}^{0} \eta^{\prime}$ in $78 \mathrm{fb}^{-1}$ of data. The measured $C P$ asymmetries and Cabibbo-suppression double ratios and are listed in Table 1.

It is in principle possible to extract $r$ from the measurements of $A_{ \pm}$and $R_{ \pm}$. However, since the $b \rightarrow c$ transition is color-allowed and and the $b \rightarrow u$ transition is colorsuppressed $r$ is likely to be small. A recent prediction gives $r \sim 0.2{ }^{47}$ The parameters $A_{ \pm}$and $R_{ \pm}$are sensitive to $r$ only in second order and thus large data samples are required to determine $r$. Due to the effect of doubly Cabibbo-suppressed $D$ decays $r$ cannot be 


\begin{tabular}{lll} 
Sample & $C P$ even & $C P$ odd \\
\hline \hline BABAR $(D K)$ & $A_{+}=0.17 \pm 0.23 \pm 0.08$ & \\
& $R_{+}=1.06 \pm 0.26 \pm 0.17$ & \\
\hline Belle $(D K)$ & $A_{+}=0.06 \pm 0.19 \pm 0.04$ & $A_{-}=-0.19 \pm 0.17 \pm 0.05$ \\
& $R_{+}=1.21 \pm 0.25 \pm 0.14$ & $R_{-}=1.41 \pm 0.27 \pm 0.15$ \\
\hline \hline
\end{tabular}

Table 1. Measured $C P$ asymmetries and Cabibbo-suppression ratios in $B \rightarrow D K$ decays.

measured from the ratio of branching fractions for $B^{+} \rightarrow D^{0} K^{+}$and $B^{+} \rightarrow \bar{D}^{0} K^{+}$ with $D$ decays to flavor specific hadronic final states. Semileptonic $D$ decays could be used for such a measurement, but large backgrounds make this approach infeasible with current data samples.

Although $r$ cannot be determined from $A_{ \pm}$and $R_{ \pm}$, from the exact relation $\left(R_{1}-\right.$ $\left.R_{2}\right) / 2=2 r \cos \delta \cos \gamma$ and the approximate relation $\left(A_{1}-A_{2}\right) / 2 \approx 2 r \sin \delta \sin \gamma$ (to order $r^{2}$ ) limits on $\gamma$ may be set. For example, from the BABAR and Belle measurements, one can extract $2 r \sin \delta \sin \gamma=0.15 \pm 0.12$ which is correct to order $r^{2}$.

An alternative method to measure $\gamma$ with $B^{+} \rightarrow D K^{+}$has been suggested to exploit the interference between the color-suppressed $b \rightarrow u$ transition followed by a Cabibboallowed hadronic $D$ decay and color-allowed $b \rightarrow c$ transition followed by a double Cabibbo-suppressed $D$ decay. ${ }^{48}$ Unfortunately, these modes are expected to have small branching fractions and have not been observed, yet.

While the extraction of the CKM angle $\gamma$ from the measured branching fractions for $B^{-} \rightarrow D K^{-}$decays is theoretically clean the current measurements indicate that about an order of magnitude more data is required to provide useful constraints for the measured $C P$ parameters.

\section{$5.2 \quad \bar{B}^{0} \rightarrow D^{(*) 0} K_{S}^{0}$}

The decays $\bar{B}^{0} \rightarrow D^{(*) 0} K_{S}^{0}$ and $B^{0} \rightarrow D^{(*) 0} K_{S}^{0}$ proceed through two color-suppressed tree amplitudes as shown in Fig. 18. The weak phase difference between the two decay amplitudes is $\gamma$ and $B^{0} \bar{B}^{0}$ mixing introduces a phase of $2 \beta$. Thus the time-dependent $C P$-asymmetries for the final states $\bar{D}^{0} K_{S}^{0}$ and $D^{0} K_{S}^{0}$ are sensitive to the parameter $2 \beta+\gamma$.

Belle ${ }^{49}$ has observed the decay $B^{0} \rightarrow \bar{D}^{0} K_{S}^{0}$ in $78 \mathrm{fb}^{-1}$ and measured its branching 

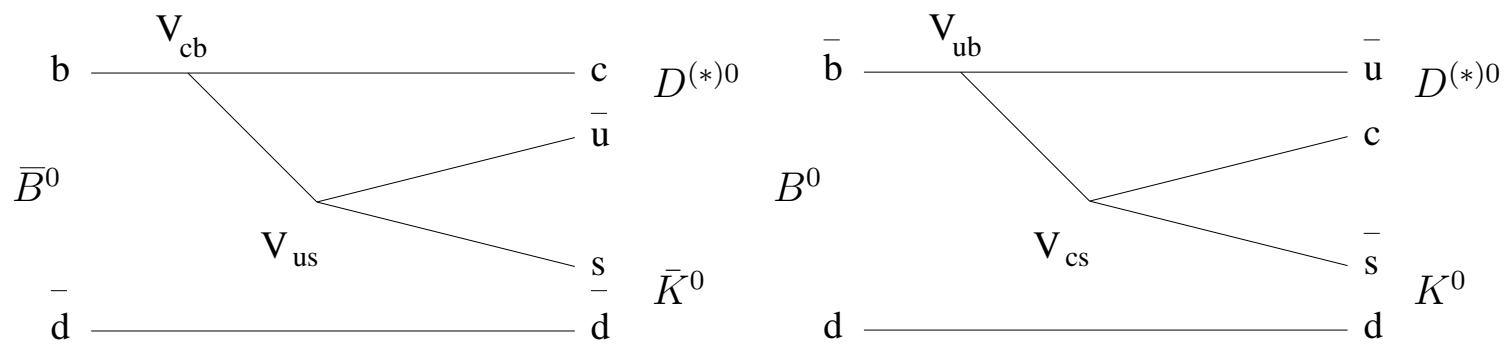

Fig. 18. Feynman-diagrams for the decay amplitudes of $\bar{B}^{0} \rightarrow D^{(*) 0} \bar{K}^{0}$ (left) and $B^{0} \rightarrow D^{(*) 0} K^{0}$ (right).

ratio to be $(5.0 \pm 1.3 \pm 0.6) \times 10^{-5}$. The $\Delta E$ distribution of the selected events is shown in Fig. 19. The selected final sample contains $27.0 \pm 7.3 B^{0} \rightarrow \bar{D}^{0} K_{S}^{0}$ events. Significantly more data is required to extract $2 \beta+\gamma$ from the time-dependence of the decay rates.

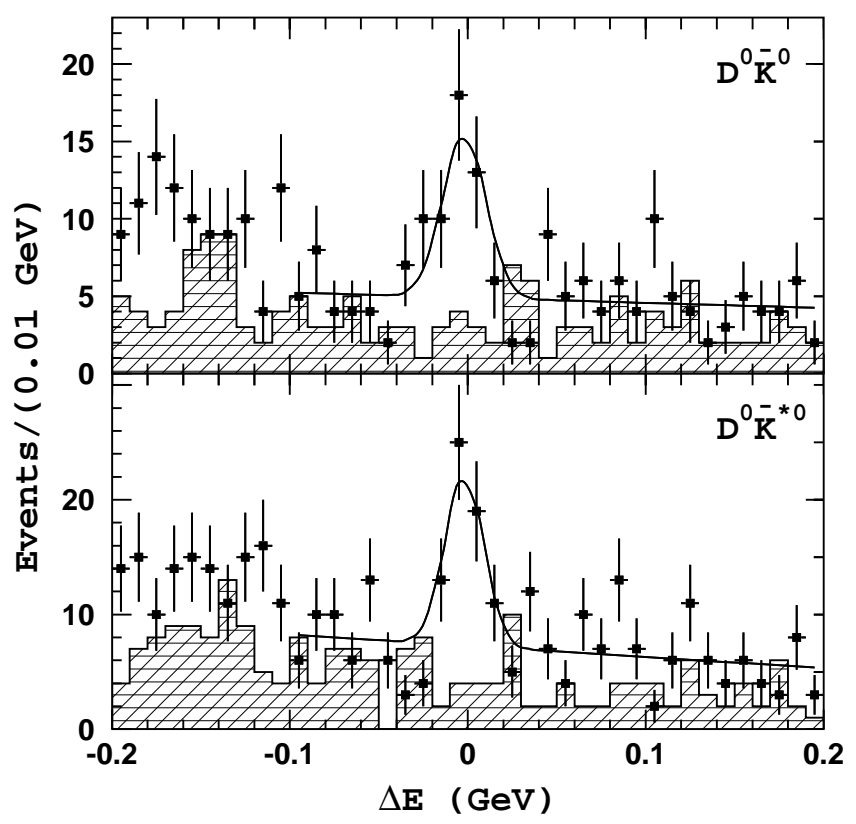

Fig. 19. Distributions of $\Delta E$ for selected $B \rightarrow D^{0} \bar{K}^{(*) 0}$ events from Belle. The hatched histogram presents events in a $D^{0}$ sideband region.

Belle also observes the (self-tagging) $b \rightarrow c$ mediated decay $B^{0} \rightarrow \bar{D}^{0} K^{* 0},\left(K^{* 0} \rightarrow\right.$ $\left.K^{+} \pi^{-}\right)$and measures its branching fraction to be $(4.8 \pm 1.1 \pm 0.5) \times 10^{-5}$. They do not observe the decay $B^{0} \rightarrow D^{0} K^{* 0}$ which proceeds through the $b \rightarrow u$ amplitude and 
set an upper limit on its branching fraction of $1.8 \times 10^{-5}$ (90\% C.L.). The ratio of the two decay rates is sensitive to the relative contributions from the $b \rightarrow c$ and $b \rightarrow u$ amplitudes. Belle also sets $90 \%$ C.L. upper limits on the branching fractions for the modes $B^{0} \rightarrow D^{* 0} K^{* 0}, B^{0} \rightarrow \bar{D}^{* 0} K^{* 0}$, and $B^{0} \rightarrow D^{* 0} K_{S}^{0}$ at $(4-7) \times 10^{-5}$.

\section{$5.3 \quad B^{0} \rightarrow D^{(*) \mp} \pi^{ \pm}$}

The decay $B^{0} \rightarrow D^{(*) \mp} \pi^{ \pm}$can proceed through a CKM-favored amplitude proportional to the CKM matrix elements $V_{c b} V_{u d}^{*}$ or through a doubly-CKM-suppressed amplitude proportional to the CKM matrix elements $V_{c d} V_{u b}^{*}$. The Feynman diagrams for both amplitudes are shown in Fig. 20. The relative weak phase between the two decay amplitudes is $\gamma$, and $2 \beta$ is introduced by $B^{0} \bar{B}^{0}$ mixing. In contrast to the $B \rightarrow D K$ decays the $V_{c b}$-mediated amplitude is less suppressed and the $V_{u b}$-mediated amplitude is more strongly suppressed. This leads to a much larger decay rate for $B^{0} \rightarrow D^{(*) \pm} \pi^{\mp}$ with a much smaller $C P$ asymmetry.

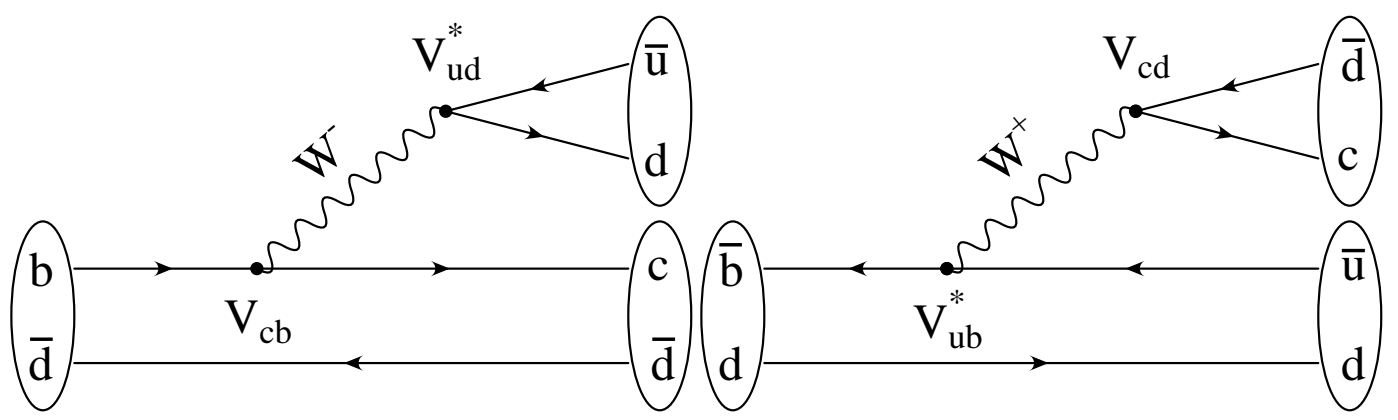

(a)

(b)

Fig. 20. Feynman diagrams for the CKM-favored decay $\bar{B}^{0} \rightarrow D^{(*)+} \pi^{-}$(left) and for the doubly-CKM-suppressed decay $B^{0} \rightarrow D^{(*)+} \pi^{-}$(right).

The decay rate distributions for $B^{0} \rightarrow D^{ \pm} \pi^{\mp}$ decays are given by

$$
\begin{aligned}
f^{ \pm}(\eta, \Delta t)= & \frac{e^{-|\Delta t| / \tau}}{4 \tau} \times \\
& {\left[1 \pm S_{\eta} \sin \left(\Delta m_{B} \Delta t\right) \mp \eta C \cos \left(\Delta m_{B} \Delta t\right)\right], }
\end{aligned}
$$

where the superscript $+(-)$ refers to whether the flavor of $B_{\operatorname{tag}}$ is $B^{0}\left(\bar{B}^{0}\right)$, with $\eta=+1$ $(-1)$ for the final state $D^{-} \pi^{+}\left(D^{+} \pi^{-}\right)$. The $S$ and $C$ parameters can be expressed as

$$
S_{\eta}=\frac{2 \mathcal{I} m\left(\lambda_{\eta}\right)}{1+\left|\lambda_{\eta}\right|^{2}}, \quad C=\frac{1-|\lambda|^{2}}{1+|\lambda|^{2}},
$$


with the definitions $|\lambda|=\left|\lambda_{+}\right|=1 / \mid \lambda_{-}$, and $\lambda_{ \pm}=\frac{q}{p} A\left(\bar{B}^{0} \rightarrow D^{\mp} \pi^{ \pm}\right) / A\left(B^{0} \rightarrow D^{\mp} \pi^{ \pm}\right)=$ $|\lambda|^{ \pm 1} e^{-i(2 \beta+\gamma \mp \delta)}$, where $\delta$ is the relative strong phase between the CKM-favored and the doubly-CKM-suppressed amplitudes. The same equations apply for $B^{0} \rightarrow D^{* \pm} \pi^{\mp}$ decays with $|\lambda|$ and $\delta$ replaced by the parameters $\left|\lambda^{*}\right|$ and $\delta^{*}$.

The measurement of $\sin (2 \beta+\gamma)$ with $B^{0} \rightarrow D^{* \pm} \pi^{\mp}$ events requires external knowledge of the ratios of the decay amplitudes $\left|\lambda^{(*)}\right|$. These ratios are expected to be small $\left(\left|V_{u b}^{*} V_{c d} / V_{u d}^{*} V_{c b}\right| \approx 0.02\right)$ and cannot be determined from $B^{0} \rightarrow D^{(*) \pm} \pi^{\mp}$ events directly with currently available data samples. However, $\left|\lambda^{(*)}\right|$ can be inferred from the ratios of branching fractions $\mathcal{B}\left(B^{0} \rightarrow D_{s}^{(*)+} \pi^{-}\right) / \mathcal{B}\left(B^{0} \rightarrow D^{(*)-} \pi^{+}\right)$using the $S U(3)$-based symmetry relation $^{50}$ :

$$
\left|\lambda^{(*)}\right|=\tan \theta_{C} \sqrt{\frac{\mathcal{B}\left(B^{0} \rightarrow D_{s}^{(*)+} \pi^{-}\right)}{\mathcal{B}\left(B^{0} \rightarrow D^{(*)-} \pi^{+}\right)}} \frac{f_{D^{(*)}}}{f_{D_{s}}(*)} .
$$

$B A B A R$ uses $|\lambda|=0.021_{-0.005}^{+0.004}$ and $\left|\lambda^{*}\right|=0.017_{-0.007}^{+0.005}$ calculated from the measurements of the Cabibbo angle $\theta_{C}$, and the branching fractions $\mathcal{B}\left(B^{0} \rightarrow D^{(*)-} \pi^{+}\right)$and $\mathcal{B}\left(B^{0} \rightarrow D_{s}^{(*)+} \pi^{-}\right)$, and from calculations of the form factor ratios $f_{D_{s}^{(*)}} / f_{D^{(*)}}$. Additional errors of $30 \%$ in $r^{(*)}$ are attributed to account for unknown theoretical uncertainties from $S U(3)$ symmetry breaking and annihilation contributions to $A\left(B^{0} \rightarrow D^{(*)+} \pi^{-}\right)$. Although a source of systematic uncertainty for all measurements of time-dependent $C P$ asymmetries in the $B^{0}$ system, given the small expected $C P$-violating amplitude in $B^{0} \rightarrow D^{*-} \pi^{+}$decays, this measurement is particularly sensitive to interference between the $b \rightarrow u$ and $b \rightarrow c$ amplitudes in $B_{\text {tag }}$ decays to certain hadronic final states. ${ }^{51}$ Therefore, events in the lepton tagging category which do not suffer from this effect contribute relatively more to these measurements than for other final states.

$B A B A R^{52}$ fully reconstructs a sample 5200 (4750) events of $B^{0} \rightarrow D^{-} \pi^{+}\left(B^{0} \rightarrow\right.$ $D^{*-} \pi^{+}$) events in $81 \mathrm{fb}^{-1}$. They also use a sample ${ }^{53}$ of partially reconstructed $B^{0} \rightarrow$ $D^{*-} \pi^{+}$events where the event topology is only determined from the (fast) pion from the $B^{0}$ decay and the (slow) pion from the $D^{*-} \rightarrow \bar{D}^{0} \pi^{-}$decay. The efficiency for partial reconstruction of $B^{0} \rightarrow D^{*-} \pi^{+}$events does not suffer from small $D^{0}$ branching ratios, but the background level is significantly higher than for fully reconstructed events.

The disributions of $m_{\mathrm{ES}}$ and $\Delta t$ of the selected $B^{0} \rightarrow D^{* \mp} \pi^{ \pm}$events are shown Fig. 21. The $C P$ parameters extracted with unbinned likelihood fits to the $\Delta t$ distributions are listed in Table 2.

From the measured $C P$ asymmetries in the fully reconstructed $B^{0} \rightarrow D^{(*)-} \pi^{+}$ events a lower limit of $|\sin (2 \beta+\gamma)|>0.69$ (68\% C.L.) is obtained. The partially 

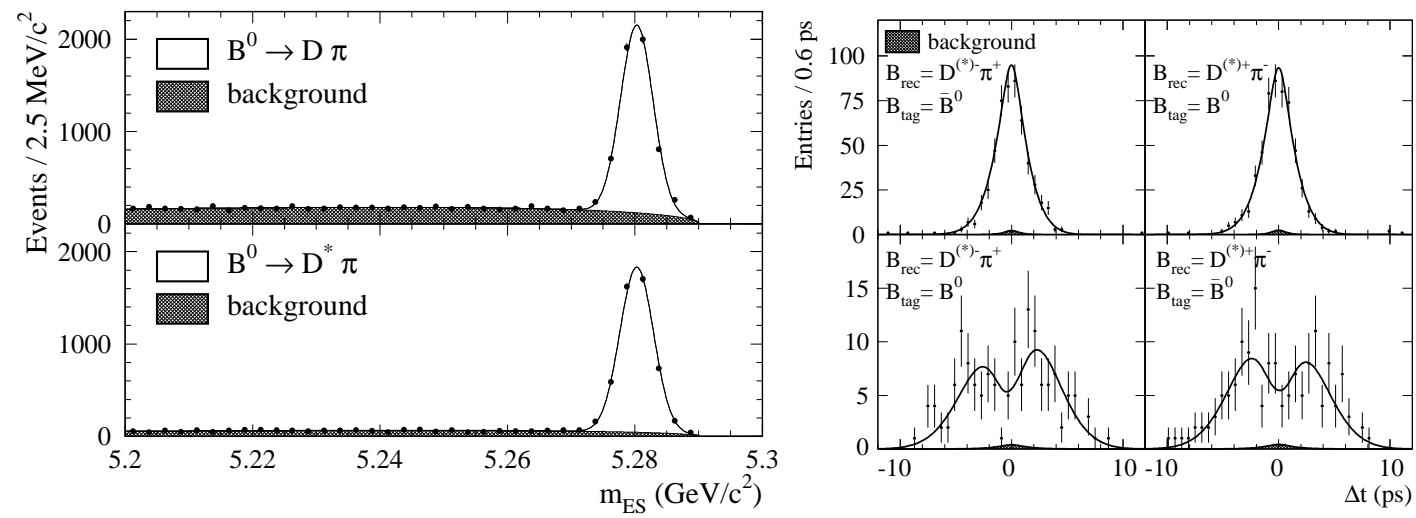

Fig. 21. Distribution of $m_{\mathrm{ES}}$ for fully reconstructed tagged $B^{0} \rightarrow D^{\mp} \pi^{ \pm}$and $B^{0} \rightarrow D^{* \mp} \pi^{ \pm}$candidates (left). Distributions of $\Delta t$ for the combined sample of fully reconstructed $B^{0} \rightarrow D^{* \mp} \pi^{ \pm}$events in the lepton tagging category (right). The solid lines present the projections of the likelihood fits and the hatched areas represent background.

reconstructed $B^{0} \rightarrow D^{*-} \pi^{+}$events yield a limit $|\sin (2 \beta+\gamma)|>0.75$ (90\% C.L.). The two samples combined give the limit $|\sin (2 \beta+\gamma)|>0.76$ (90\% C.L.).

\begin{tabular}{lcc} 
Sample & $2\left|r^{(*)}\right| \sin (2 \beta+\gamma) \cos \delta$ & $2\left|r^{(*)}\right| \cos (2 \beta+\gamma) \sin \delta$ \\
\hline$B^{0} \rightarrow D^{ \pm} \pi^{\mp}$ & $-0.02 \pm 0.04 \pm 0.02$ & $0.03 \pm 0.07 \pm 0.04$ \\
$B^{0} \rightarrow D^{* \pm} \pi^{\mp}$ (fully reco.) & $-0.07 \pm 0.04 \pm 0.02$ & $0.03 \pm 0.07 \pm 0.04$ \\
$B^{0} \rightarrow D^{* \pm} \pi^{\mp}$ (part. reco.) & $-0.063 \pm 0.024 \pm 0.017$ & $-0.004 \pm 0.037 \pm 0.020$ \\
\hline
\end{tabular}

Table 2. BABAR measurements of the time-dependent $C P$ asymmetries in $B^{0} \rightarrow$ $D^{(*) \mp} \pi^{ \pm}$events.

\section{Conclusion}

The $B$ factories $B A B A R$ and Belle have established $C P$ violation in the $B$ meson system. The measurement of $\sin 2 \beta$ with charmonium kaon final states is now more than thirteen standard deviations different from zero and has become the most precise constraint on the apex of the Unitarity Triangle. It is interesting to note that the measurement of $\sin 2 \beta$ with penguin-dominated modes differs by about $2.5 \sigma$ from this measurement. More data will show if this is just a statistical fluctuation or the first evidence for $C P$ violation from physics beyond the standard model. The Unitarity Triangle angles $\alpha$ and $\gamma$ have 
still much larger theoretical and experimental uncertainties. For the determination of $\alpha$ with $B^{0} \rightarrow \pi^{+} \pi^{-}$and $B^{0} \rightarrow \rho^{ \pm} \pi^{\mp}$ a better understanding of the contribution of the penguin amplitudes is needed. On the other hand the $B \rightarrow D K$ modes are theoretically clean, but much larger statistics is required for a precise determination of $\gamma$.

The measurements from $B A B A R$ and Belle have not disproved the CKM prediction of one complex phase as single source of $C P$ violation, yet. However, as said in the beginning, the current measurements of $C P$ asymmetries are unlikely to explain the matter anti-matter asymmetry in the universe. Thus, to further exclude the possibility of $C P$ violation in the quark mixing matrix beyond the CKM prediction BABAR and Belle will accumulate more than $500 \mathrm{fb}^{-1}$ per experiment over the next few years and continue to improve the precision on the Unitarity Triangle parameters.

\section{References}

[1] YA.D. Sakharov, ZhETF Pis. Red. 5, 32 (1967); JETP Lett. 5, 24 (1956).

[2] P. Langacker in "CP Violation” edited by C. Jarlskog, World Scientific (1989).

[3] W. Bernreuther, Lect.Notes Phys. 591, 237 (2002).

[4] J.H. Christenson et al. Phys. Rev. Lett. 13, 138 (1964).

[5] N. Cabibbo, Phys. Rev. Lett. 10, 531 (1963);

M. Kobayashi and T. Maskawa, Prog. Th. Phys. 49, 652 (1973).

[6] L. Wolfenstein, Phys. Rev. Lett. 51, 1945 (1983).

[7] A.B. Carter and A.I. Sanda, Phys. Rev. D23, 1567 (1981);

I.I. Bigi and A.I. Sanda, Nucl. Phys. B193, 85 (1981).

[8] BABAR Collaboration, B. Aubert et al., Nucl. Instrum. Methods. A479, 1, (2002).

[9] Belle Collaboration, A. Abashian et al., Nucl. Instrum. Methods. A479, 117, (2002).

[10] BABAR Collaboration, B. Aubert et al., Phys. Rev. Lett. 89, 201802 (2002).

[11] Belle Collaboration, K. Abe et al., Phys. Rev. D 66, 071102 (2002).

[12] BABAR Collaboration, B. Aubert et al. Phys. Rev. Lett. 87, 241801 (2001);

Belle Collaboration, K. Abe et al. Phys. Lett. B538, 11 (2002).

[13] Heavy Flavor Averaging Group,

http://www.slac.stanford.edu/xorg/hfag/triangle/winter2003/ 
[14] A. Hoecker, H. Lacker, S. Laplace, F. LeDiberder, Eur.Phys.Journ. C21, 225 (2001), updated results and plots at http://ckmfitter.in2p3.fr.

[15] BABAR Collaboration, B. Aubert et al., Phys. Rev. Lett. 91, 061802 (2003).

[16] Belle Collaboration, K. Abe et al., hep-ex/0207098.

[17] Y. Grossman and M. Worah, Phys. Lett. B 395, 241 (1997).

[18] M. Gronau, Phys. Lett. B 300, 163 (1993).

[19] BABAR Collaboration, B. Aubert et al., Phys. Rev. Lett. 90, 221801 (2003).

[20] M. Gronau, Phys. Rev. Lett. 63, 1451 (1989).

[21] A.I. Sanda and Z.Z. Xing, Phys. Rev. D 56, 341 (1997).

[22] X.Y. Pham and Z.Z. Xing, Phys. Lett. B 458, 375 (1999).

[23] J. Rosner, Phys. Rev. D 42, 3732 (1990).

[24] Z.Z. Xing, Phys. Rev. D 61, 014010 (2000).

[25] BABAR Collaboration, B. Aubert et al., Phys. Rev. Lett. 91, 131801 (2003).

[26] Y. Grossman, G. Isidori, M.P. Worah, Phys. Rev. D 58, 057504 (1998).

[27] G. Hamel De Monchenault, BABAR Collaboration, hep-ex/0305055.

[28] Belle Collaboration, K. Abe et al., Phys. Rev. D 67, 031102 (2003).

[29] D. London and A. Soni, Phys. Lett. B 407, 61 (1997);

M. Beneke and M. Neubert, Nucl. Phys. B 651, 225 (2003).

[30] BABAR Collaboration, B. Aubert et al., Phys. Rev. Lett. 91, 161801 (2003).

[31] Belle Collaboration, K. Abe et al., Phys. Rev. D 67, 031102 (2003).

[32] M. Beneke, G. Buchalla, M. Neubert, and C.T. Sachrajda, Nucl. Phys. B 606, 245 (2001); Y.Y. Keum, H-n. Li, and A.I. Sanda, Phys. Rev. D 63, 054008 (2001); M. Ciuchini et al., Phys. Lett. B 515, 33 (2001).

[33] BABAR Collaboration, B. Aubert et al., Phys. Rev. Lett. 89, 281802 (2002).

[34] Belle Collaboration, K. Abe et al., Phys. Rev. D 68, 012001 (2003).

[35] H. Sagawa, hep-ex/0307060.

[36] M. Gronau and D. London, Phys. Rev. Lett. 65, 3381 (1990).

[37] BABAR Collaboration, B. Aubert et al., Phys. Rev. Lett. 91, 241801 (2003).

[38] Belle Collaboration, S.H. Lee et al. Phys. Rev. Lett. 91, 261801 (2003). 
[39] Y. Grossman and H.R. Quinn, Phys. Rev. D 58, 017504 (1998).

[40] M. Gronau, D. London, N. Sinha, and R. Sinha, Phys. Lett. B514, 315 (2001).

[41] A. Höcker, H. Lacker, M. Pivk, and L. Roos, hep-ph/0307013.

[42] BABAR Collaboration, B. Aubert et al., Phys. Rev. Lett. 91, 201802 (2003).

[43] M. Gronau and D. Wyler, Phys. Lett. B265 172 (1991);

M. Gronau and D. London, Phys. Lett. B253 483 (1991).

[44] The BABAR physics book, editors P.F. Harrison and H.R. Quinn, SLAC-R-504 (1998).

[45] BABAR Collaboration, B.Aubert et al., hep-ex/0207087.

[46] Belle Collaboration, S.K. Swain et al., Phys. Rev. D 68, 051101 (2003).

[47] M. Gronau, Phys. Lett. B557, 198 (2003).

[48] D. Atwood, I.Dunietz, and A. Soni, Phys. Rev. Lett. 78, 3257 (1997).

[49] Belle Collaboration, P. Krokovny et al., Phys. Rev. Lett. 90, 141802 (2003).

[50] I. Dunietz, Phys. Lett. B427, 179 1998); I. Dunietz and R.G. Sachs, Phys. Rev. D 37, 3186 (1988); R. Fleischer, hep-ph/0304027.

[51] O. Long, M. Baak, R.N. Cahn, D. Kirkby, Phys. Rev. D 68, 034010 (2003).

[52] BABAR Collaboration, B.Aubert et al., hep-ex/0309017.

[53] BABAR Collaboration, B.Aubert et al., hep-ex/0310037. 